\title{
Die Verweise Leonardo da Vincis auf seine verlorenen Traktate zur Mechanik*
}

\author{
VON DIETRICH LOHRMANN
}

\section{Überblick}

Mit den Verweisen Leonardos auf eigene, guten Teils verlorene Werke befasst sich die Wissenschaft beiläufig seit spätestens 1870, Klarheit hat sie nicht erreicht. Auch von den 120 eigenen Schriften, die Leonardo gegen Ende seines Lebens mit dem Bedauern ansprach, sie nicht vollendet zu haben, sind umfangreiche Fragmente in einem bisher nur schwer zu bestimmenden Umfang erhalten. Es fehlt eine Übersicht über diese Titel und eine Zusammenstellung der Fragmente, die eine Zuweisung zu den einzelnen Titeln erlauben. In Abschnitt 2 ist das Problem anhand der einschlägigen Verweise aus Leonardos Codex Madrid I (1493-1495) nur vorläufig angesprochen. Auf die Benutzung antiker Autoren (Abschnitt 3) wird vor allem hingewiesen, um eine Typologie der Verweise in ihrer Verschiedenheit abzubilden. Es zeigt sich, dass die wichtigsten antiken Autoren bei der Arbeit am Codex Madrid I noch nicht zur Verfügung standen: Euklid wurde erst ab 1195/96 für Leonardo wichtig, Archimedes noch später.

Als weiteres verlorenes Werk kommt hinzu der seit langem bekannte Titel der Elementi macchinali (Abschnitt 4). Die jetzt 16 Verweise auf dieses Werk stammen aus deutlich späteren Handschriften Leonardos. Codex Atlanticus fol. 421v liefert eine (geplante) Inhaltsübersicht in mindestens 20 Abschnitten. Die Intention war eine Systematisierung des reichen Inhalts von Madrid I mit Schwerpunkt auf der Statik (Entstehung ca. 1495-1499). Eine Rekonstruktion zumindest der Grundstrukturen dieses Werkes mit den in Madrid I angezeigten Textstellen scheint möglich. In Teil 5 geht es um ein verlorenes Werk über Statik und Dynamik, ein Werk, das mindestens neun Teile umfasste. Sein Titel war vermutlich teorica meccanica. Der Codex Madrid I liefert für dieses frühe Werk 47 Verweise, verteilt auf beide Teile des Bandes. Das sich aus ihnen ergebende verlorene Werk liegt damit chronologisch vor 1493 bis 1495.

* Für seine effiziente Unterstützung bei der Ermittlung und Transkription der Belegstellen danke ich Frank Hasters, Aachen. Mannigfache Anregungen vermittelten Dr. Ulrich Alertz und Dr. Thomas Kreft. Alle drei sind Mitarbeiter am DFG-Projekt „Leonardo da Vinci, Codex Madrid I. Kommentierte Neuausgabe“, durchgeführt am Historischen Institut der RWTH Aachen (vgl. Anm. 10). Freundliche Beratung verdanke ich außerdem Gerhard Dohrn van Rossum, Chemnitz, und Marcus Popplow, Heidelberg. Eine verkürzte englische Fassung dieses Beitrags ist in den Archives internationales d'histoire des sciences geplant. 


\begin{abstract}
Since at least 1870 , studies on Leonardo da Vinci mention his numerous references to lost works on mechanical subjects. The titles invoked give a good idea of how intensive was Leonardo's engagement. An autograph note written at the end of his life speaks of 120 writings and regrets that there would not be time to complete all of them. Even now, there is still no inventory of such titles, no typology of forms, no method to allow the allocation of titles to fragments conserved in the manuscripts.

Section 2 gives some relevant references from the core of Codex Madrid I (1493-1495), but much work remains concerning these titles. Section 3 draws attention to Euclid's Geometrical elements; references to this work are all posterior to the genesis of Codex Madrid I.

Part 4 and 5 of this paper are focused on the reconstruction of two lost works in which Leonardo tried to present systematically his views on medieval statics and aspects of late medieval dynamics. The first one, a work in nine books, contained three parts on dynamics. For this early work Madrid I provides 47 references. The second lost work, mentioned only in later manuscripts, deals with the long known title of Elementi macchinali (Section 4). There are now 16 references. For this work we have also a preparatory index of matters offered by the Codex Atlanticus which mentions 22 sections. We have to add Codex Madrid I fol. $82 \mathrm{r}$, a first summoning up of matters to be treated in the future Elementi macchinali. The intention was a systemization of the rich but chaotic contents of Madrid I. This inventory of rules and proofs must have been written between 1495-96 and 1499. It is lost but can be reconstructed in a good part of its essential sectors.
\end{abstract}

$$
* * *
$$

Wie wohl bekannt ist, bezeichnete sich Leonardo da Vinci gelegentlich selbst als einen Menschen ohne literarische Bildung, als uomo senza lettere. In seiner Kindheit war ihm keine höhere Schulbildung zuteil geworden. Nach den Forschungen Marinonis fehlte ihm bis etwa zum 40. Lebensjahr jede genauere Kenntnis in Latein und Mathematik. ${ }^{1}$ Trotzdem entwickelte er sich seit den 1490er Jahren zum Verfasser umfangreicher wissenschaftlicher Ausarbeitungen, insbesondere auch solcher zur Mechanik. Wie ein akademischer Gelehrter scheute er sich dabei nicht, eifrig auf eigene Schriften wie auf solche anderer Autoren zu verweisen.

Gelehrte Verweise auf Stellen des eigenen Werkes oder solche anderer Autoren waren in der wissenschaftlichen Literatur des späteren Mittelalters längst zur gängigen Praxis geworden. Ein erheblicher Schub im Bereich der

1 Augusto Marinoni, La matematica di Leonardo da Vinci. Una nuova immagine dell'artista scienziato, Mailand 1982, S. 18-29, 60-73. 
mathematisch-mechanischen Studien ging seit dem 12. bis 13. Jahrhundert von den Elementen des Euklid aus. Das war bekanntlich eines der einflussreichsten Werke der mathematischen Literatur und wurde ab 1495 auch durch Leonardo da Vinci mit Eifer benutzt. Die Verweise hatten bei Euklid Beweisfunktion wie etwa 3.26: „Es folgt nach [Satz] 19 dieses [Buches], dass b und f gleich sind und umgekehrt" (,,sequitur b et $\mathbf{f}$ esse equales per 19 huius et $\mathrm{e}$ converso"). Busards neue Edition der Euklid-Bearbeitung durch Campanus von Novara (ca. 1256) macht die Praxis mehr als deutlich. ${ }^{2}$ Jordanus Nemorarius (13. Jh.), ebenfalls einflussreich als Mathematiker und Leonardo früh bekannt, benutzte gleichermaßen Verweise auf Euklid wie solche auf ein verlorenes eigenes Werk, den Philotegni. ${ }^{3}$ Häufig erscheinen sie im Albion, einem bedeutenden astronomischen Traktat des Richard von Wallingford (1326): „Durch den 4. [Satz] dieses [Teils] findet man“ („Per 4am prime huius inveniatur"). ${ }^{4}$ Leonardo bekannt, da italienisch geschrieben, war wohl auch der große Kommentar des Lorenzo Ghiberti (um 1450) zur Perspektive, wo eine Fülle von Verweisen auf ältere Werke zur Optik zu finden ist. Und ganz ähnlich wie bei Leonardo da Vinci (unten Abschnitt 5) begnügt sich dessen bedeutender Nachfolger Guidobaldo dal Monte in seinem systematischen Mechaniktraktat von 1577 (lat.) bzw. 1581 (ital.) mit knappen Zahlenverweisen: ,per la 18. del primo, per la 3 . del terzo“ und so vielfältig. ${ }^{5}$

Die hier näher zu beobachtende Praxis Leonardos steht somit in einer dauerhaft etablierten Tradition. Die Vielfalt seiner Verweisziele und der verschiedenen Verweisformen macht die Sache bei ihm allerdings kompliziert. Wie er in vieler Hinsicht als Autodidakt für sich selbst schreibt, so verweist er auch für sich selbst und scheut sich dabei nicht, selbst ungeschriebene, nur intendierte oder kaum begonnene Werke zu benennen.

2 H.L.L. Busard, Campanus of Novara and Euclid's Elements I (Boethius 51, 1), Stuttgart 2005, S. 114ff.; zur Datierung vgl. ebd., S. 32.

3 Ernest A. Moody u. Marshall Clagett, The Medieval Science of Weights (Scientia de Ponderibus), Madison, Wisconsin 1960, S. 119-227. Vgl. Fritz Schuster, Zur Mechanik Leonardo da Vincis (Hebelgesetz, Rolle, Tragfähigkeit von Ständern und Trägern), Diss. Erlangen 1915, S. 26. Die nützliche Arbeit stammt aus der Schule des Erlanger Physikers und Arabisten Eilhard Wiedemann.

4 Richard of Wallingford, Tractatus Albionis (hg. von John D. North), Oxford 1976, Bd. 1, S. 304.

5 Zu Ghiberti vgl. die genauen Nachweise auf Euklid, Ptolemäus, Alhazen, Roger Bacon u.a. bei Klaus Bergdolt, Der dritte Kommentar Lorenzo Ghibertis, Weinheim 1986; Guidobaldo del Monte, Le mechaniche, Venezia 1581 (lat.: Mechanicorum libri sex, 1577). Das Werk wurde 1629 ins Deutsche übersetzt. Der lange Titel dieser Übersetzung ist bemerkenswert: „Mechanischer Kunst-Kammer Erster Theil. Von Wag, Hebel, Scheiben, Haspel, Keil und Schrauffenwerckh. Darinn der wahre unfehlbare Grund aller künstlicher und sinnreicher Machination begriffen etc., mit ausführlichen Kupfer-Figuren zu vollkommenem Verstand vor augen gebildet durch Danielem Möglin, Wirtemb. der Artzney Doctorn, Landgräf-Hessischen Hoff-Medicum und Mathematicum zu Putzbach in der Wetterau, Frankfurt am Main in Verlegung Matthaei Merian MDCXXIX“. 
Die Verweise Leonardos auf eigene, scheinbar verlorene Schriften bilden seit langem ein schwer zu lösendes Problem. Als Giambattista Venturi 1797 vor der französischen Akademie erstmals Einblick in Leonardos naturwissenschaftliche Arbeiten gewährte, sprach er die Frage noch nicht an. Er konzentrierte sich auf die 14 Skizzenhefte, die im Auftrag Napoleons zusammen mit dem Codex Atlanticus von Mailand nach Paris gelangt waren, und wollte vor allem deren Inhalt erschließen. Immerhin bemerkte er schon, es handle sich in diesen Heften um Gelegenheitsnotizen, der Autor habe sie nachfolgend zu vollständigen Traktaten ausarbeiten wollen. ${ }^{6}$

Im 19. Jahrhundert wandte man sich näher der Deutung von Leonardos Traktat über die Malerei zu, dem einzigen Werk Leonardos, das schon im 17. Jahrhundert gedruckt erscheinen konnte. Dabei stieß man auf zahlreiche Titel scheinbar verlorener Werke und stellte sich die Frage nach dem Verbleib dieser Arbeiten. Seitdem ist mit dem Bekanntwerden weiterer Leonardo-Handschriften die Zahl solcher Verweise ständig angewachsen. Ihre Deutung fällt nach wie vor schwer. Sicher ist nur: Es sind in jedem Fall aufschlussreiche und unentbehrliche Zeugnisse für eine genauere Kenntnis von Leonardos Arbeit an einer eindringenden Analyse der mechanischen Probleme.

Mit den beiden erst 1965 wieder entdeckten Codices Madrid I und II kamen besonders zahlreiche Verweise auf eigene Sätze und Darlegungen hinzu. ${ }^{7}$ Vor allem der erste Band, datiert im Kern 1493 bis $1495,{ }^{8}$ nach dem Inhalt vornehmlich Studien zu Maschinenelementen und mechanischer Theorie, enthält vielerlei Hinweise dieser Art. Die Frage nach dem genauen Ziel und Sinn dieser Verweise verschärfte sich, sie wurde nun auch offen angesprochen. ${ }^{9}$ Will man verstehen, welches Werk jeweils gemeint ist, und dies in der

6 Giovanni Battista Venturi, Essai sur les ouvrages physico-mathématiques de Léonard de Vinci, Paris 1797, S. 4 (ND LaVergne, TN USA 2010). Vor Venturi hat 1704 schon der große italienische Historiker Ludovico Muratori einen Teil der Handschriften Leonardos gesehen und treffend bemerkt, dass es vor allem unvollendete Fragmente seien: ,preparazioni dell'autore per opere ideate all'ordine". Giuseppe Campori, Lettere artistiche inedite, Modena 1866, S. 534-542. Vgl. Arturo Uccelli, Leonardo da Vinci. I libri di meccanica, Mailand 1940, S. XXXII. Die zwölf Pariser Skizzenhefte wurden zuerst herausgegeben durch Charles Ravaisson-Mollien, 6 Bde., Paris 1881-1891. Sie sind als Facsimilia Teil der Edizione nazionale des Verlags Giunti, Florenz, hg. durch Augusto Marinoni, 12 Bde., 1986-1990, und sind seit ca. 2008 im Internet zugänglich durch das Programm e-leo der Biblioteca comunale in Vinci; Einen Überblick über das Gesamtwerk Leonardos als Maler, Zeichner und Autor handschriftlicher Aufzeichnungen vermittelt der Band von Simona Cremante, Leonardo da Vinci, Florenz, Mailand 2005. Dem dortigen Verzeichnis der Manuskripte (S. 485-635) entnehme ich die Datierungsansätze für die Entstehung der einzelnen Handschriften. Die wichtigsten Datierungsansätze eruierte schon Gerolamo Calvi, I manoscritti di Leonardo da Vinci dal punto di vista cronologico, storico e biografico, Mailand 1975 (Neuedition durch Augusto Marinoni, 1982).

7 Ladislao Reti (Hg.), Leonardo da Vinci, Codices Madrid I-II, 5 Bde., deutsche Ausgabe Frankfurt 1974.

8 Zur Datierung des Codex Madrid I vgl. ebd., Bd. 3, S. 43.

9 Vgl. das Folgende, insbes. Anm. 26 u. 60. 
derzeit in Arbeit befindlichen „Kommentierten Internet-Edition“ des Codex Madrid I näher erläutern, ${ }^{10}$ bleibt keine andere Wahl, als zunächst ein möglichst breites Spektrum von Leonardos Verweisen zusammenzustellen und $\mathrm{zu}$ versuchen, sie typologisch zu differenzieren.

Eine solche Sucharbeit war vor wenigen Jahren noch praktisch undurchführbar. Sie wird heute nachhaltig unterstützt durch die Möglichkeiten der elektronischen Abfrage. Die Voraussetzung dafür erfüllt ein rasch fortschreitendes Unternehmen mit Namen Leonardo digitale bzw. e-leo, betreut von der Stadtbibliothek Vinci mit Unterstützung der Europäischen Union. In ihm sind alle wichtigen Handschriften zur Mechanik Leonardos mittlerweile zugänglich. ${ }^{11}$

Am Schluss unserer Untersuchung geht es um zwei verlorene Werke Leonardos. Eines über Maschinenelemente (Elementi macchinali), auf das in der Literatur schon vielfältig hingewiesen wurde, das andere bisher ganz unbekannt. Drei Bemerkungen sind dabei schon jetzt wesentlich: 1) Die Zahl der bisher bekannten direkten Verweise auf die Elementi macchinali war bisher klein und muss nach Möglichkeit vermehrt werden. 2) Im Codex Madrid I von etwa 1493 bis 1495 (Nachträge bis etwa 1499) erscheinen Hinweise auf dieses Werk noch nicht. 3) Stattdessen erscheinen in dieser Handschrift sehr zahlreiche präzise Kurzverweise auf ein unbekanntes, systematisch gegliedertes Werk, das dem Autor des Codex Madrid I bereits zitierfähig vorlag, aber ebenfalls verloren ist.

\section{Das Problem in der älteren Leonardo-Literatur}

Die älteste Beschäftigung mit Leonardos Selbstzitaten in der deutschsprachigen Leonardo-Literatur geht zurück auf den Kunsthistoriker Max Jordan. Sie betrifft Leonardos postum veröffentlichte Schrift über die Malerei (De pittura). Jordan hat die Titel bereits 1872 bis 1873 zusammengestellt und eine wichtige Unterscheidung vorgenommen: „Es ist zu scheiden zwischen solchen, die er mit der Zahl und solchen, die er mit Titel nennt". Aufgeführt werden die Bücher I, II, III, IV, VI, IX und X mit Ziffern, offensichtlich alles Hinweise auf Bücher des Trattato della pittura, sowie Hinweise auf andere Werke mit Titeln wie, Schatten und Licht (Obra e lume), Perspektive (Prospettiva), Bücher über die verschiedenen Formen von Bewegungen (Libro de 'movimenti, Nr. 10, Libro de 'moti, Nr. 11), ein Buch über Moto locale im aristotelischen Sinn (Nr. 12), sowie weitere Bücher über Anatomie (Nr. 13),

10 Leonardo da Vinci, Codex Madrid I. Kommentierte Internet-Edition, DFG-Projekt RWTH Aachen, Historisches Institut (in Arbeit). Vgl. Dietrich Lohrmann, Warum eine Neuedition des Codex Madrid I von Leonardo da Vinci?, in: Technikgeschichte 76, 2009, S. 131-146.

$11 \mathrm{Zu}$ e-leo bzw. Leonardo digitale vgl. Anm. 6 und kurz erläuternd Marcus Popplow, Die Rückkehr des Künstler-Ingenieurs. Tendenzen und Perspektiven der Forschung zu Leonardo da Vinci, in: NTM 16, 2008, S. 131-144. Näheres in einem Essay unserer künftigen Internet-Edition. 
über die mittelalterliche Statik (De ponderibus, Nr. 14), über die Universal misura dell'uomo (Nr. 15), die Piegamenti dell'uomo (Nr. 16), über den Flug der Vögel (Uccelli, Nr. 17), über Elementi allgemein (Nr. 18). ${ }^{12}$

Max Jordan kannte mindestens 18 Werke Leonardos, während dieser selbst gegen Ende seines Lebens von insgesamt 120 Büchern bzw. Titeln spricht, nur die fehlende Zeit habe ihn daran gehindert, sie abzuschließen. ${ }^{13}$ Neben anderen Schriften erkennt man in Jordans Aufzählung unschwer sein nicht abgeschlossenes Werk über den Vogelflug (Uccelli), das inzwischen in verschiedenen Formen rekonstruiert worden ist. ${ }^{14}$ Außerdem klärte Jordan im Anschluss an Leonardos Freund und Lehrer in der Mathematik, Luca Pacioli (ca. 1447-1517), dass bestimmte unter den genannten Werken Leonardos im Jahre 1498 bereits vollendet waren, so das Werk über die Malerei und das über die menschlichen Bewegungen. Hingegen habe er seine Abhandlungen über die Ortsbewegung (Moto locale) und über Stoß und Gewicht der Kräfte (De le percussioni e pesi de le force) zu diesem Zeitpunkt noch ,unter der Feder" gehabt. ${ }^{15}$

1874, angeregt durch Franz Reuleaux, den großen Systematiker der kinematischen Mechanik, spricht Hermann Grothe die Verweise ebenfalls an, in seiner immer noch lesenswerten Studie über Leonardos technische Schriften. Er beschränkt sich freilich auf die Anzeige eigener Schriften des Autors und nennt keine Fundstellen. Namentlich kennt er ein Buch von der Bewegung (Libro del moto), einen Traktat vom Stoß (Trattato di percussione), ein Buch vom Antrieb bzw. Schwung (Libro del impeto), ein Buch von der Schwere (Libro di gravità) und ein Werk über die Grundlagen der Mechanik (Elementi macchinali). „Solche Schriften“, schreibt er, „scheinen in Form von Leitfäden angelegt worden zu sein, nach $\S \S$ geordnet, auf die Leonardo

12 Max Jordan, Das Malerbuch des Leonardo da Vinci, Habilitationsschrift Leipzig 1872, S. 26. Dazu Übersicht über die Verweise des Libro della pittura im Anhang derselben Arbeit, die vollständig nur in Zahn's Jahrbuch der Kunstwissenschaft 5, 1873, S. 273-370, erschienen ist (hier S. 331-334).

13 „Cento 20 libri da me composti ne daran sententia [...], nelle quali non sono stato impedito nè d'avaritia o negligentia, ma sol dal tempo", zitiert von Uccelli (wie Anm. 6), S. XIII nach den Quaderni d'Anatomia in Windsor. Kurz zuvor auf einem späten Blatt des Codex Atlanticus, fol. 463v erwähnt Leonardo sein 113. Werk: „E con queste si compone l'ultima mia opera di cento 13 libri da me composti“. Die hier benutzte Edition ist Augusto Marinoni u. Carlo Pedretti (Hg.), Leonardo da Vinci, Il codice Atlantico della Biblioteca Ambrosiana di Milano, 3 Bde., Florenz 2000, hier Bd. 2, S. 875.

$14 \mathrm{Zu}$ den älteren Ausgaben des Codice del volo degli uccelli seit 1893 vgl. die Angaben bei Marianne Schneider, Leonardo da Vinci, Der Vögel Flug - Sul volo degli uccelli, München 2000, S. 17. Wichtig insbesondere die kommentierte Ausgabe von Arturo Uccelli, Mailand 1952, die auch die Textauszüge berücksichtigt, die den Turiner Codex ergänzen, und Domenico Laurenza, Leonardo. Il volo, Florenz, Mailand 2004.

15 Jordan (wie Anm. 12), S. 18, nach Pacioli, De divina proportione Tract. I, Brief Paciolis an Herzog Lodovico Moro vom 9.2.1498: ,avendo già con tutta diligentia al degno libro de pictura e movimenti humani posto fine". 
dann in gewissen Fällen einfach hinweist“. Ein Zitat hebt Grothe hervor: "Questa è manifesta per la dodicesima, e provasi ancora per l'ottava che dice [...]“ (,Dies wird deutlich durch Satz 12 und lässt sich beweisen durch Satz 8 , der besagt $\left.[\ldots]^{6}\right)$. Ferner nennt Grothe ein Londoner Manuskript (gemeint vermutlich der Codex Arundel), in dem eine Abhandlung über Wellenbewegung, den Moto ondoso, enthalten sei. Dort fänden sich Zitate, die sich auf eine vorhandene Zusammenstellung der hydrostatischen Gesetze bezögen. ${ }^{16}$

1913 veröffentlichte Franz Maria Feldhaus eine Studie über Leonardo als Techniker und Erfinder mit vielen guten Beschreibungen einzelner Mechanismen bzw. Maschinen. Auch ihm sind die Verweise auf andere Schriften aufgefallen. $\mathrm{Zu}$ seinen Lebzeiten ließ Leonardo von all den vielen technischen Arbeiten nichts im Druck erscheinen. Seine Aufzeichnungen machte er entweder in ganz kleine Notizbücher, in kladdenförmige Schreibhefte, oder auf einzelne Notizblätter ganz verschiedenen Formats. Manche dieser Aufzeichnungen hatten anscheinend eine einheitliche Anordnung. So spricht Leonardo z.B. gelegentlich von seinem ,Buch vom Stoß`, vom ,Buch von der Schwere', vom Buch vom ,Kraftmoment' oder von seinem ,Buch über die Maschinenelemente'. Keines dieser Bücher, bemerkt Feldhaus, habe sich erhalten. Manches unter den erhaltenen Heften handele wenigstens durchweg von dem gleichen Gegenstand, so z. B. ein in russischem Besitz befindliches ,Buch über den Vogelflug'. ${ }^{6}$

\section{Verweise auf andere Schriften Leonardos mit Titel}

Die weiteren Erwähnungen von Leonardos Verweisen in der gelehrten Literatur des früheren 20. Jahrhunderts übergehen wir. ${ }^{18}$ Man kannte das Problem, kam aber nicht wesentlich weiter und wechselte deshalb die Methode: Weg vom Nachweis bestimmter verlorener Titel bzw. Werke, statt dessen logisch-inhaltliche Rekonstruktion. Prominentester Vertreter dieser neuen Konzeption war (1940) der große Sammler von Leonardos Einzelaussagen zu den verschiedensten Themenbereichen seiner mechanischen Studien,

16 Hermann Grothe, Leonardo da Vinci als Ingenieur und Philosoph, Berlin 1874 (ND Leipzig o.J.), S. 35; Marianne Schneider (Hg.), Leonardo da Vinci, Das Wasserbuch. Schriften und Zeichnungen, München 1996, S. 17f. (Gliederung aus Arundel, fol. 35r, 45r), S. 77 87 und bes. S. 36 (Kapitel über Wellen und Wirbel, wo aber nur ein Satz aus dem Codex Arundel zitiert wird).

17 Franz Maria Feldhaus, Leonardo der Techniker und Erfinder, Jena 1913, S. 3. Das ,Buch über den Vogelflug' lag ursprünglich als Teil des Ms. B in Mailand. Wie die anderen Pariser Skizzenbücher gelangte es 1796 nach Paris. Dort entwendete es der bekannte Astronomie- und Mathematikhistoriker Guiglielmo Libri um 1840. Auf verschlungenen Wegen gelangte es, weiter aufgespalten, über Russland (Theodor Sabachnikov) nach Turin. Vgl. Laurenza (wie Anm. 14), S. 85.

18 Wichtig wären die uns noch nicht zugänglichen Arbeiten von Roberto Marcolongo 1934 und 1939. Károly Simonyi spricht in seiner Kulturgeschichte der Physik, Leipzig 1990, S. 166 von einem Tagebuch Leonardos, in dem er ,nur zu unzusammenhängenden Skizzen gelangt" sei. 


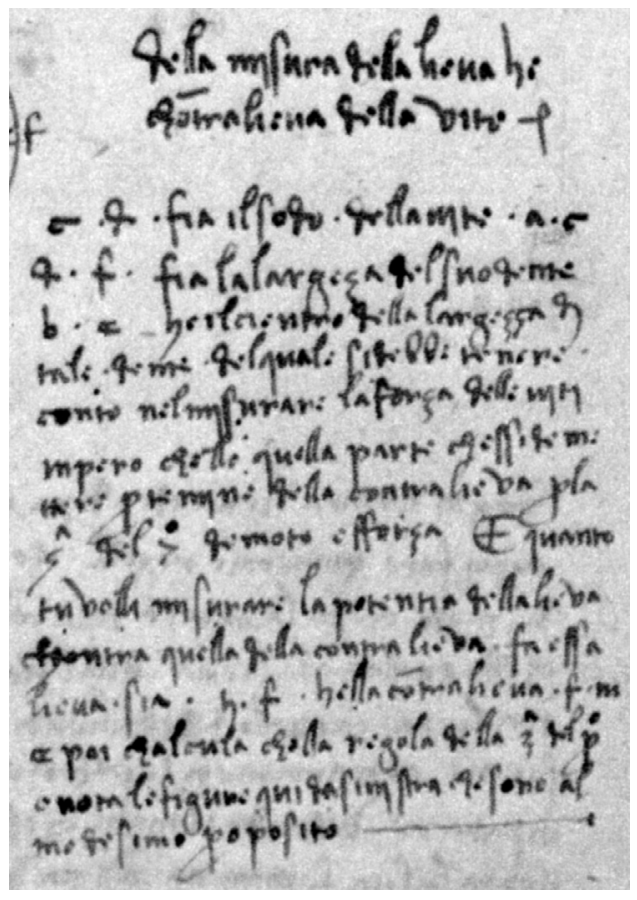

Abb. 1: Codex Madrid I fol. 94r (gespiegelt): „Inpero ch' ell' è quella parte che ssi de' mettere per termine della contralieva, per la 5 a del $7^{\circ}$ de moto e fforza. $[\ldots]$ E poi calcola colla regola della $3^{\circ} \mathrm{del}$ primo."

Arturo Uccelli. ${ }^{19}$ In einer gewaltigen Kompilationsarbeit vereinte er aus sämtlichen damals bekannten Leonardo-Handschriften die verfügbaren Aussagen Leonardos zur Mechanik in 17 Abschnitten und füllte damit nicht weniger als 463 dicht bedruckte Seiten. Seine Anordnung erfolgt ausschließlich thematisch, bewusst nicht chronologisch, da viele Darlegungen bzw. Handschriften schlecht datiert seien. Seine Kriterien der Zuweisung an die jeweiligen Abschnitte sind nicht immer leicht nachzuvollziehen. In jedem Falle handelt es sich um Einheiten, die so erst Arturo Uccelli gebildet hat. ${ }^{20}$

$\mathrm{Zu}$ den einzelnen Verweisen Leonardos, die Uccelli in großer, hier noch keineswegs darstellbarer Fülle, wenn auch verstreut an weit auseinander liegenden Stellen, wiedergibt, vermerkte er wiederholt, dass es nicht möglich sei, beim gegenwärtigen Erhaltungszustand der Schriften Leonardos einen genauen Bezugspunkt für sie anzugeben. ${ }^{21}$ Darin sind ihm viele gefolgt.

19 Uccelli (wie Anm. 6).

20 Vgl. Arturo Uccelli, Ricostruzione della meccanica vinciana. Necessità e discussione del metodo, in: Raccolta vinciana 15, Mailand 1935-1939, S. 22-63.

21 Uccelli (wie Anm. 6), S. 171, Anm. 2; S. 234, Anm. 1; S. 235, Anm. 1; S. 243, Anm. 2; S. 256, Anm. 1; S. 294, Anm. 1; S. 352, Anm. 1; S. 356, Anm. 1; S. 390, Anm. 3 etc. Auf S. 365, Anm. 1 notiert Uccelli zu einem Verweis „4 del 7“ im Codex Forster II, 2: „intende forse alludere al libro degli ,Elementi macchinali“, was nach den Ergebnissen unserer nachfolgenden Abschnitte nicht möglich ist. Forster II, 2 fällt etwa in dieselbe Zeit wie Madrid I. Der Verweis geht demnach auf das verlorene frühere Werk in neun Teilen, mit dem wir uns im Abschnitt 5 beschäftigen. 
Insgesamt jedoch brachte das Werk Uccellis 1940 einen enormen inhaltlichen Fortschritt in der Kenntnis der Forschungen Leonardos zur Mechanik. Neben 17 so genannten Libri di meccanica ,rekonstruierte" er vier weitere Bücher unter dem speziellen Titel Elementi macchinali. Zu dem letzten Werk konnte er freilich nur einen Verweis anführen, in dem Leonardo ausdrücklich diesen Obertitel nennt, während sich dank der elektronischen Suchfunktion der Internet-Edition e-leo inzwischen ca. zwölf solche Verweise gefunden haben und weitere noch hinzugekommen sind. ${ }^{22}$

Eine neue Situation ergab sich mit der Wiederentdeckung der beiden Codices Madrid I und II ab 1965/66. Der Ertrag aus Madrid I spiegelt sich schon im Index des dritten Bandes von Retis Edition 1974. ${ }^{23}$ Wir stellen hinzu die entsprechenden Abschnitte der Rekonstruktionen Uccellis, übergehen dagegen die 1974 in der Übersetzung und im Index ergänzten Angaben „Abschnitt“ und „Kapitel“, wenn ihre Deutung unsicher ist. Der Originaltext gibt jeweils nur Ordnungszahlen in weiblicher Form für die Unterabschnitte: ,5a“, „,7a“ etc. (,sentenzia“, ,,regola“ oder ähnliches), und maskuline Formen für den Oberabschnitt (,libro“ oder „,capitolo“): ${ }^{24}$

fol. 0v (Deckblatt): „Buch über Quantität und Potenz“ („Libro titulato de quantità e potentia“). Mit dieser Angabe meinte Leonardo schwerlich seine unmittelbar nachfolgende Ausarbeitung im Codex Madrid I, obwohl der ,Potentia'-Begriff dort häufig vorkommt. Der Stoff erscheint aber nicht geordnet und vereint als Buch. Zudem steht der Eintrag auf fol. 0v isoliert neben einer nachgetragenen Gelegenheitsnotiz vom 28. September 1497. Möglicherweise hat Leonardo damals an dem Thema erneut gearbeitet, z.B. im Rahmen der verlorenen Elementi

22 Uccelli (wie Anm. 6), S. 465. Zu den Verweisen auf die Elementi macchinali vgl. unten Abschnitt 4. Der großen Zahl weiterer, bei Uccelli aus anderen Handschriften zitierter Einzelwerke nachzugehen, war noch nicht möglich. Hier wartet eine reiche Ernte. Nur drei Beispiele seien angemerkt: 1. Libro primo delle acque (Codex Arundel, fol. 266r; Uccelli [wie Anm. 6], S. 29; Ed. Pedretti-Vecce 1998: S. 106f., S. 129 u. S. 133). Entwürfe zu diesem lang geplanten Werk finden sich u.a. in den Codices Leicester, fol. 15r und Arundel, fol. 45r. Vorgesehen waren danach 15 bzw. 16 als libri bezeichnete Kapitel. Weiteres bei Edward McCurdy, The Notebooks of Leonardo da Vinci 2, London 1938, S. 78, S. 96-99 u. S. 105 (mit anderen Bücherzahlen); bei Theodor Lücke (Hg.), Leonardo da Vinci, Tagebücher und Aufzeichnungen, Leipzig 1940, S. 528 (F fol. 24v-25r) und Schneider (wie Anm. 16), S. 17-19. 2. Libro de confregatione (Codex Atlanticus, fol. 1043r): „E di questo si tratterà nel libro de confregazione“. Umfangreiche Materialien zu diesem geplanten Buch über Reibung bei Uccelli (wie Anm. 6), S. 355-383. Das Thema beschäftigte Leonardo schon im Ms. Paris A, fol. 132-134 (ca. 1490-1492) und erscheint mehrfach auch im Codex Madrid I; vgl. Uccelli (wie Anm. 6), S. 259-353. 3. Libro 43 del moto dell'aria inclusa sotto l'acqua (Paris Ms. F, fol. 37b); J.P. Richter, The Notebooks of Leonardo da Vinci, London 1883 (ND 1970, 2, S. 394, n. 1338). Offensichtlich Teil eines großen geplanten Werkes De moto mit über 40 Teilen bzw. Büchern.

23 Reti (wie Anm. 7), Bd. 3, S. 140f. Entsprechend lauten die Angaben in den gleichzeitig erschienenen Ausgaben in englischer, spanischer, französischer und italienischer Sprache.

24 Vgl. unten Abschnitt 5. 
macchinali, in denen er Madrid I als Vorlage benutzte (keine direkte Entsprechung bei Uccelli [wie Anm. 6]).

fol. 69r: ,5. vom 3. von Bewegung und Schlag“ (,per la 3a del $5^{\circ}$ di moto e colpo“). Der Abschnitt handelt von Stoß und Fallrichtung des Wassers in Mühlen (vgl. Uccelli [wie Anm. 6], S. 399-463 [De percussione]).

fol. 69r: „Vorletztes über Bewegung und Widerstand“ (,penultima di moto e resistentia“) (vgl. Madrid I, fol. 95r [De moto e resistentia]).

fol. 70v: „Über die Veränderung der Lage Buch 3“ (,Della mutatione de’ siti libro 3“). Von der Struktur dieses Werkes ist bisher wenig Sicheres bekannt. Gemeint scheint unter anderem die Lage der Gewichte auf dem Waagbalken und die Theorie von der ,gravitas secundum situm', also Statik.

fol. 84v: „Abhandlung über das (geführte) Ziehen“ („De stracinatione“) (vgl. die Materialien zum Thema ,De’ semplici tirari e alzari‘ bei Uccelli [wie Anm. 6], S. 469-491).

fol. 94r: „5. des 7. über Bewegung und $\mathrm{Kraft}^{\prime \prime}$ (,5a del $7^{\circ}$, de moto e fforza“). Es geht um die Mitte der Gewindehöhe einer Schraube, die als Ende des Gegenhebels anzunehmen sei: „Inperoch' ell' è quella parte che ssi de' mettere per termine della contralieva“" (vgl. Uccelli [wie Anm. 6], S. 305-332).

fol. 97v: ,7. des 4. über Kraft und Widerstand“ (,per la 7a del $4^{\circ}$, de potentia e resistentia“" (vgl. Madrid I, fol. 95r [,potentia e resistentia“], 117r [,De potentia e resistentia']). Dieser vierte Teil kann zu dem unten Abschnitt 5 behandelten Werk gehört haben.

fol. 103r: „8. des Buchs über den Antrieb“ (,Questa 8a è allegata nel Libro dell'inpeto. Adunque queste figure vanno in esso libro“ (vgl. Uccelli [wie Anm. 6], S. 385398 [De impeto]).

fol. 139r: Abhandlung über Mathematik (ein geplantes Werk, wie das Futurum zeigt): „,nel mio trattato delle matematiche si dirà distintamente ongni prova e ragion precisamente".

fol. 140v: ,7. des 9. der Theorie“ (,,per la 5a de $7^{\circ}$ della teorica“) (vgl. unten Abschnitt 5, fol. 147v: ,5. (Abschnitt) meiner Theorie“).

Es folgen im Index Retis 32 Kurzverweise des Typs „3. des 1.“ oder ,5. des 1." ohne Angabe eines Titels. Auf sie gehen wir im Abschnitt 5 dieser Untersuchung näher ein.

Etwa gleichzeitig wie Reti besprach 1974 Carlo Zammattio in einer Arbeit über Leonardos naturwissenschaftliche Studien die Erwähnung eigener Werke. Er bezeichnete sie als ,geplante Bücher über Mechanik“. Zu Leonardos Gewölbestatik bemerkt er, hier habe er sich an Regeln und Lehrsätze der Mechanik gehalten, die er ,,in die Form eines systematischen Corpus von theoretischen Erkenntnissen" brachte. Die erhaltenen Leonardo-Manuskripte ergäben kein klares Bild, in welchem Sinn diese Ausarbeitungen gedacht waren, als Sammlung von Regeln oder als nach und nach ausgearbeitete Notizen. „Jedenfalls“, fährt Zammatio fort, ,gibt es verschiedene Listen mit 
Überschriften der von Leonardo geplanten Bücher über Mechanik.“ Die Hinweise auf Kapitelnummern und Lehrsätze in seinen Untersuchungen seien „so genau, als bezögen sie sich auf eine schon abgeschlossene Zusammenstellung". 25

Ausführlicher erörtert die Verweise des Codex Madrid I eine amerikanische Romanistin in ihrer Dissertation, die sie 2000 an der Universität von Pennsylvania abschloss. ${ }^{26}$ Da sie sich insbesondere den kurzen Selbstzitaten Leonardos zuwendet, verweisen wir auch hier auf Abschnitt 5. Von den neueren Stellungnahmen sei nur noch das Werk des amerikanischen Mechanikprofessors Francis C. Moon berücksichtigt. Er behandelt die Entwicklung der Maschinenkinematik ausgehend von Leonardo da Vinci bis zu Franz Reuleaux. Zu fol. 17r des Codex Madrid I bemerkt er: „This last line of Leonardo's seems to indicate that he had in mind a scheme for classification of mechanisms according to the type of motion transformation produced by the device. This program for kinematics of machines was formalized by faculty at the Ecole Polytechnique in Paris in the late $18^{\text {th }}$ century “ ${ }^{27}$

\section{Verweise auf Euklids Elemente und andere antike Autoren}

Bevor wir auf die für uns wichtigsten Gruppen der Verweise Leonardos eingehen, seien seine Hinweise auf antike Autoren eingeschoben. Am eindeutigsten sind die etwa 30 Verweise auf Euklids Elemente der Geometrie. Keiner von ihnen findet sich im Codex Madrid I. Durch Madrid II hingegen sind etliche bisher unbekannte Euklid-Verweise hinzugekommen. Damit steht im Einklang ein Ergebnis von Augusto Marinoni. Nach seinen Untersuchungen kann sich Leonardo mit Euklid erst ab 1495 beschäftigt haben. Frühestens Ende 1494 kaufte er sich ein soeben erschienenes Buch des Mathematikers Luca Pacioli und begann mit dessen Hilfe, als etwa 42-Jähriger, seine Lücken in der Arithmetik und Geometrie aufzuarbeiten. ${ }^{28}$

Dieser Vorgang intensivierte sich noch erheblich, als Luca Pacioli 1496 selbst nach Mailand kam, öffentlich Vorlesungen über die Elemente des Euklid anbot und Leonardo in unmittelbaren Kontakt zu ihm trat. Marinoni hat diesen Vorgang minutiös nachvollzogen und kommt zu dem Ergebnis, dass nur die Hilfe des Mathematikers es Leonardo erlaubt haben kann, den Text Euklids

25 Carlo Zammattio, Naturwissenschaftliche Studien, in: Ladislao Reti (Hg.), Leonardo Forscher, Künstler, Magier, Frankfurt 1974, S. 190-215, insb. S. 212. Vgl. ders., Leonardo der Forscher, Darmstadt 1981, S. 58.

26 Marina della Putta Johnston, Leonardo da Vinci's Codex Madrid I: The Creation of the Self as Author, Diss. University of Pennsylvania 2000, S. 47-50.

27 Francis C. Moon, The Machines of Leonardo da Vinci and Franz Reuleaux. Kinematics of Machines from the Renaissance to the 20th Century, Dordrecht 2007, S. 324.

28 Kauf von Luca Pacioli, Summa de arithmetica, Venedig 1494, zum Preis von 119 Soldi: Cod. Atlanticus fol. 288r (alt 104r-a). Auszüge Leonardos aus Paciolis Summa de aritmetica (De multiplicatione fractorum) finden sich im Codex Atlanticus, fol. 192v-191r (rückläufig), vgl. die Vorbemerkungen bei Marinoni/Pedretti (wie Anm. 13), Bd. 1, S. 253-260. 


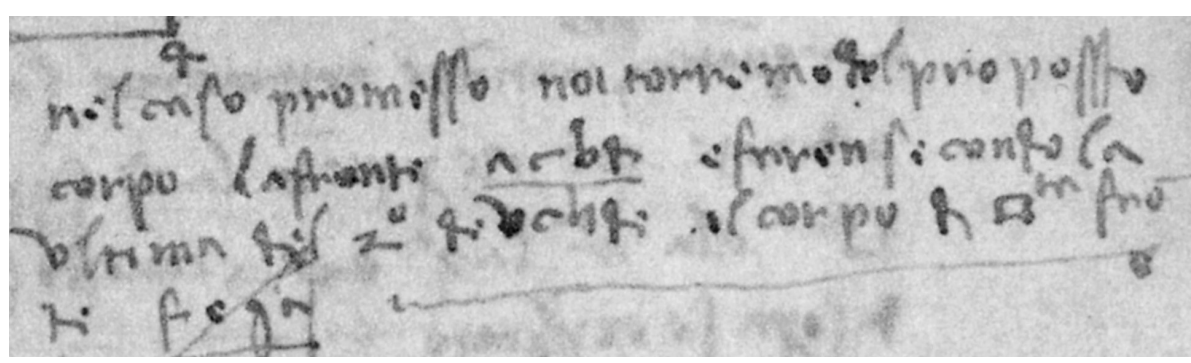

Abb. 2: Codex Madrid II fol. 50v (gespiegelt): „Nel caso promesso noi torremo del propossto corpo la fronte $\mathbf{a} \mathbf{c} \mathbf{b} \mathbf{d}$ e faren, seconda la ultima del $2^{\circ} \mathrm{d}$ 'Euclide, il corpo di quadrata fronte f e g a."

in der Ausgabe des Campanus von Novara (ca. 1256) zu verstehen. Die Begegnung mit Luca Pacioli fällt in die Jahre 1496 bis $1499 .{ }^{29}$ Hier folgen zunächst sieben ausgewählte Beispiele für Verweise auf Euklid:

\section{Euklid Verweis Wortlaut}

1, letzter Madrid II „Provasi coll'ortogonio a $\mathbf{c} \mathbf{b}$, che per l'ultima del primo [Satz] 120v degli Elementi geometrici, il lato a c può lui solo quanto i' lato b c e '1 lato a b.“",Mit dem rechtwinkligen [Dreieck] $\mathbf{a} \mathbf{c} \mathbf{b}$ wird bewiesen, dass die Seite $\mathbf{a} \mathbf{c}$ entsprechend dem letzten des ersten der Geometrischen Elemente allein soviel vermag wie die Seiten $\mathbf{b} \mathbf{c}$ und $\mathbf{a}$ b“. (Gemeint ist der berühmte Satz des Pythagoras, bei Euklid Buch I, Kap. 46-47 [Ausgabe des Campanus von Novara]).

1,40 Paris E „A b c d sia il dato quadrato, el quale s'abbi a astendere 10 $\mathrm{r} \quad$ in [...]. Per la quadragesima del primo delli Elementi io porroò il dato quadrato $\mathbf{a} \mathbf{b} \mathbf{c} \mathbf{d}$ e continuerò la linia $\mathbf{b} \mathbf{f}$, equale alla linia $\mathbf{d}$ b.“. „Nach dem 40. des 1 . der Elemente setze ich das gegebene Quadrat a b c d und ziehe die Linie b f weiter, gleich der Linie $\mathbf{d} \mathbf{b}$." (Das Alter der Handschrift E [1513-15] und die Übereinstimmung mit der Herkunft des folgenden Verweises sprechen für Euklid I, 41 [Parallelogramm]).

1, $40 \quad$ Paris E $\quad,[\ldots]$ fatto il quadrilatero $\mathrm{K} \mathbf{i} \mathbf{h}$ a equale al quadrato dato 10r a b c d, e questo si prova mediante la quadragesima del primo d'Euclide.“, ,[...] ist das Viereck $\mathbf{K} \mathbf{i}$ h a gleich dem Quadrat a b c d, und das wird bewiesen mit dem 40. des ersten von Euklid.“

29 Marinoni (wie Anm. 1), S. 73-88. 
2, letzter Madrid II „Nel caso promesso noi torremo del propossto corpo la Abschnitt 50v fronte a b c d e faren, secondo la ultima del secondo d'Euclide, il corpo di quadrata fronte f g e a.“ „Im genannten Fall nehmen wir von dem genannten Körper die Stirnseite a b c d weg und bilden, entsprechend dem letzten des zweiten von Euklid, einen Körper mit quadratischer Stirnseite f g e a." (Zeichnung im Faksimileband Retis, fol. 50v, zweite Reihe). Es geht Leonardo bereits um Stereometrie, Euklid nur um Flächen.

Atl. 607r „Li quali quadrati lunghi si riducano in quadrati perfetti con una delli Elementi, cioè questa [Figura].“ „Diese langen Quadrate werden zu vollkommenen Quadraten entsprechend einer [Skizze] der Elemente, d.h. dieser [Figur]“ (vgl. Marinoni/Pedretti [wie Anm. 13], S. 1186, Anm. 7 [Bezug auf Euklid]).

Atl. $607 \mathrm{r} \quad$,e in $4758 \mathrm{mi}$ dà la quantità della accresciuta basa del diminuito cilindro, a la quale per una d'Euclide i' crescerò il lato b a.“ „und in 4758 gibt er mir die Größe der vergrößerten Basis des verkleinerten Zylinders, dessen Seite b a ich entsprechend einem [Satz] des Euklid vergrößern werde." (Pedretti gibt zu dieser Seite eine ausführliche Erklärung).

Paris K „Quinto Euclide. Prima definizione.“ - „Fünftes [Buch $51 \mathrm{r}$ des] Euklid. Erste Definition“

Von diesen sieben Verweisen aus Codex Madrid II, Codex Atlanticus und den Codices Paris E und K nennen vier Euklid direkt, einer betont ausdrücklich die geometrischen Elemente, die beiden übrigen stehen in euklidischen Zusammenhängen, so dass eine Zugehörigkeit zu Leonardos allgemeinerem, von Uccelli rekonstruierten Traktat über die Elemente Luft, Feuer, Wasser und Erde ausgeschlossen erscheint. Die Handschrift Paris K stammt von 1503 bis 1507, sie beweist bisher als einzige einen Rückgriff Leonardos auf das im westlichen Mittelalter vor 1150 noch unbekannte Buch 5 von Euklids Elementen. Uccelli nennt 24 weitere Euklid-Verweise in anderen Handschriften Leonardos; die Verweise aus dem Codex Madrid II kannte er noch nicht. ${ }^{30}$ Vor allem im Codex Forster I von ca. 1505 finden sich diese Hinweise; sie beziehen auch die stereometrischen Körper (Zylinder, Kubus) bei Euklid ein. Zwei unserer Beispiele behandeln ebenfalls solche Körper.

Im Codex Madrid II fol. 140v bis 138v finden sich zudem - aus heutiger Sicht rückläufig transkribiert - die ersten Seiten einer italienischen Überset-

30 Uccelli (wie Anm. 6), S. LXXX. 
zung von Euklids Elementen, die vermutlich auf Luca Pacioli zurückgeht und von Leonardo benutzt wurde. ${ }^{31}$ Vor der Wiederentdeckung von Madrid II kannte man eine italienische Übersetzung erst aus dem Druck durch Nicola Tartaglia, Venedig 1543. Für einen Mathematikhistoriker wäre es vermutlich eine interessante Aufgabe zu untersuchen, in welcher Weise Leonardo die insgesamt etwa 30 Stellen des Euklid genutzt hat.

Euklids Geometrie erscheint auch in der jüngeren der beiden Bücherlisten Leonardos, die etwa 1503 bis 1505 entstand: Codex Madrid II, fol. 2v-3r (insgesamt 116 Titel). Nr. 23 dieser Liste nennt Euclide in geometria, Nr. 110 Euclide vulgare, cioè e primi libri 3. Man darf annehmen, dass Leonardo sein Studium des Euklid mit der italienischen bzw. toskanischen Übersetzung begonnen hat; möglicherweise erhielt er sie, wie schon bemerkt, durch Luca Pacioli. ${ }^{32}$ Unter den 40 Titeln der älteren Bücherliste im Codex Atlanticus fol. 559r (alt fol. 210 r.a) ist Euklid (im Gegensatz zu Plinius) noch nicht verzeichnet.

Das sehr kleine Notizbuch Forster III von etwa 1493 bis 1496 verzeichnet auf fol. 2v einen bemerkenswerten Hinweis auf Euklids Statik: Der Arzt Magister Stefano Caponi wohne beim Schwimmbad, er besitze Euklids Werk De ponderibus. ${ }^{33}$ Am ehesten entspricht dieser Titel einem pseudo-euklidischen Werk De ponderoso et levi, das seit dem 13. Jahrhundert aus Handschriften in Venedig und Dresden bekannt ist. Eine vermutlich echte Überlieferung in arabischer Übersetzung war Leonardo nicht bekannt, sie ist erst 1851 durch Ferdinand Woepcke herausgegeben worden. ${ }^{34}$

Sehr großen Einfluss auf Leonardos Anschauungen zur Dynamik im Bereich der Mechanik hatten die naturphilosophischen Schriften des Aristoteles. Als direktes Zitat aus dessen Physik in lateinischer Sprache kennen wir bisher nur Atlanticus fol. 226v (83 v-b): „Si debeat motus esse, oportet quod prius sit mobile et motivum. In $2^{\circ}$ de $8^{\circ}$ Fisicorum“. Vor allem die dynamischen Prozesse sind angesprochen. Hier steht Leonardo ganz in der Tradition der spätmittelalterlichen Physik, die sich bekanntlich von Aristoteles nur sehr schwer lösen konnte. Arturo Uccelli und Marshall Clagett zeigen, wie die alten Vorstellungen von den Elementen, vom Schweren und Leichten, vom Vakuum, vom natürlichen Ort und vielem anderen bei Leonardo vor allem durch die aristotelisch geprägte Naturphilosophie der Scholastiker beinflusst wurden. ${ }^{35}$

31 Vgl. dazu Augusto Marinoni, Leonardos Schriften, in: Reti (wie Anm. 25), S. 56-85, hier S. 66.

32 Ladislao Reti, The Library of Leonardo da Vinci, Los Angeles 1972, S. 10 und 26. Vgl. Reti/Marinoni zu Leonardos Bibliothek in der Ausgabe der Codices Madrid von 1974, Bd. 3, S. 101, 114. Lateinische Ausgaben der Elemente Euklids lagen seit 1482 vor, sie beruhten u.a. auf der Übersetzung des Campanus de Novara, vgl. Busard (wie Anm. 2). „Maestro Stefano Caponi medico sta alla Piscina: ha Euclide de Ponderibus“.

34 Näheres bei Uccelli (wie Anm. 6), S. LXXX-LXXXIII.

35 Ebd., S. XXXV-LXIV; Marshall Clagett, Dictionary of Scientific Biography 7, 1970, S. 215-225. 
Uccelli zufolge leitete Leonardo einige Leitsätze auch aus den antiken, dem Aristoteles zugeschriebenen Problemata mechanica ab (1497 erstmals gedruckt). ${ }^{36}$ Uccelli vorauf gingen die bahnbrechenden Untersuchungen von Pierre Duhem. Er bewies im Bereich der Statik die Neuansätze der Schule des Jordanus Nemorarius (13. Jh.), die Leonardo ebenfalls intensiv rezipiert hat, auch sie erst nach Anfertigung italienischer Auszüge aus der mittelalterlichen Scientia ponderum. ${ }^{37}$

Auf Archimedes deutet in Madrid I direkt noch nichts, mit Ausnahme von Ausführungen über den Schwerpunkt ebener Flächen und den von Körpern (u.a. fol. 164r bis 166v). Man wird manche andere theoretische Aussage aber mit solchen in späteren Arbeiten Leonardos vergleichen müssen. Bei den Schwerpunkten sind dies insbesondere die Angaben im Codex Arundel. Nach Clagett finden sich in den späteren Leonardo-Handschriften Spuren von fünf berühmten Werken des Archimedes: 1. Kreismessung, 2. Spirallinien, 3. Kugel und Zylinder, 4. Gleichgewicht und Schwerpunkt ebener Flächen sowie 5. Schwimmkörper.

Leonardo bezieht sich außerdem auf einzelne Handschriften von Werken des Archimedes, so insbesondere auf die Archimedes-Handschrift des Bischofs von Padua und eine Handschrift in Urbino. Paris L fol. 2r (ca. 15021504) berichtet dazu: „Borges [Antoine Boyer, Erzbischof von Bourges] wird dir den Archimedes des Bischofs von Padua besorgen und Vitellozzo [Condottiere des Cesare Borgia] den aus Borgo San Sepolcro“. Codex Atlanticus fol. 968 b zeigt Leonardo geradezu als Handschriftenjäger, der detektivisch Nachrichten über den Verbleib der vollständigen Archimedes-Handschrift aus Urbino nachspürt: „Archimenide è intero appresso al fratel di monsignore di santa Giusta in Roma. Disse averlo dato al fratello che sta in Sardigna. Era prima nella libreria del duca d'Urbino; fu tolto al tempo del duca Valentino“. Mit dem Herzog von Valencia war Cesare Borgia gemeint. Der Eintrag stammt demnach aus der Zeit nach dessen Sturz im Oktober 1503. ${ }^{38}$

$\mathrm{Zu}$ den historischen Angaben im Zusammenhang mit Archimedes gehört die bekannte Aussage zur Dampfkanone: „Architronito è una macchina di

36 Uccelli (wie Anm. 6), S. 305f. Erstdruck der dem Aristoteles zugeschriebenen Problemata mechanica: Venedig (Aldus) 1497 im vierten Band der Opera omnia graece, cum Theophrasti operibus nonnullis. Vgl. Aristotele, Problemi meccanici, a cura di Maria Elisabetta Bottecchia Dehò, Catanzaro 2000, S. 12, Anm. 32.

37 Pierre Duhem, Les origines de la statique, 3 Bde., Paris 1905-1906 (engl. 1991) ; ders., Études sur Léonard de Vinci. Ceux qu'il a lus et ceux qui l'ont lu, 3 Bde., Paris 19051913 (ND 1984). Italienische Auszüge aus der Scientia ponderum hat der Codex Atlanticus f. 416v-r, 449r u. 981r. Vgl. die Hinweise Pedrettis in Marinoni/Pedretti (wie Anm. 13), Bd. 2, S. 764f.

38 Weiteres über diese Handschriften hat A. Favaro, Archimede e Leonardo da Vinci, in: Atti del reale Istituto Veneto di Scienze, Lettere ed Arti 71, 1911-1912, S. 953-975. Identifizierung mit den erhaltenen Handschriften bei Marshall Clagett, Leonardo da Vinci and Archimedes, in: ders., Archimedes in the Middle Ages III, 3, Philadelphia 1978, S. $477-$ 523 , bes. S. 479. 
fine rame, invenzione d'Archimede". ${ }^{39}$ Aus der frühen italienischen Übersetzung der Berühmten Männer des Plutarch stammt wahrscheinlich die Nachricht: „fu d'Archimede siracusano, il quale in quel tempo dimorava in compagnia di Ecliderides, re de' Cirodastri““ ${ }^{40}$ Im Codex Atlanticus fol. $413 \mathrm{v}-$ 413r findet sich ein Auszug aus Archimedes De insidentibus in humido (Schwimmkörper). Die Schrift dieser Kopie stammt nicht von Leonardo. ${ }^{41}$

Uccelli kennt darüber hinaus einen singulären Verweis auf Heron von Alexandrien („Erone de acque“) im Codex Atlanticus fol. 264v (alt $96 \mathrm{v}-\mathrm{a}$ ), wiederholt auf fol. 589v (alt 219v-a). In den Pariser Notizbüchern F fol. 1v (Messer Atavian Palavisino pel suo Vetruvio), G fol. 96v und I fol. 53v erscheint ferner der Name des Vitruv, auf den eine von Leonardos berühmtesten Zeichnungen zurückgeht. ${ }^{42}$ Schließlich erwähnt der wissbegierige Leonardo 1489 in einer frühen terminologischen Zusammenstellung zu antiken Waffen (Paris B fol. 43v) neben Varro und Pompeius (Trogus?) auch die Kommentare des Julius Caesar. ${ }^{43}$

Wichtiger für den Kommentar zum Codex Madrid I ist Plinius der Ältere. Leonardo kannte seine Historia naturalis durch die italienische Übersetzung des Cristoforo Landino von 1476. Nach Roberto Cardini 1973 lieferte schon Edmondo Solmi 1908 ,la dimostrazione del larghissimo uso che Leonardo fece del Plinio landiniano". ${ }^{44}$ Besonders interessant im Codex Madrid I fol. 112r ist der Vorschlag einer technischen Lösung für die bei Plinius erwähnte Vereinigung zweier halbkreisförmiger antiker Theater zum Vollkreis eines Amphitheaters. Um die beiden Theatertribünen zu bewegen, benutzte Leonardo breite Rollen, die in der spätmittelalterlichen Technik beliebt waren; auf ihnen drehten sich unter anderem die Hauben der spätmittelalterlichen Turmwindmühlen. Ganz am Anfang des Codex Madrid I (fol. 0r) erwähnt Leonardo schließlich, wohl in Anlehnung an Plinius oder Plutarch, den systematischen Kunstraub der Römer in den von ihnen eroberten Ländern. ${ }^{45}$

39 Paris ms. B, fol. 33; vgl. dazu Theodor Beck, Beiträge zur Geschichte des Maschinenbaues, Berlin 1899, S. 348f.; Ladislao Reti, Il mistero dell'Architronito, in: Raccolta Vinciana 19, 1962, S. 171-183.

40 Paris BnF, ms. 2037, fol. 9; Uccelli (wie Anm. 6), S. LXV-LVI; vgl. Clagett (wie Anm. 38), S. 480 (historical references).

41 Codex Atlanticus, fol. 413rv; vgl. Clagett (wie Anm. 38), S. 513-515 (floating bodies).

42 Uccelli (wie Anm. 6), S. LXXVI-LXXIX u. S. 466.

43 „Una asta molto acuta, la quale in forma di telo ovver dardo si po con mano trarre, secondo Varrone e Pompeo e Cesere nel $5^{\circ}$ de' Commentari“. Leonardo benutzte hier ein Wörterbuch unter dem Stichwort, tragula'.

44 Roberto Cardini, La critica del Landino, Florenz 1973, S. 165. E. Solmi, Le fonti dei manoscritti di Leonardo da Vinci, Turin 1908 (Neudruck Florenz 1976), S. 235-248 (Auszüge aus Plinius, Buch VIII Zoologie).

45 Nähere Angaben im künftigen Kommentar zu Madrid I, fol. 112r u. 0r. 


\section{Leonardos verlorene Elementi macchinali (1495-1499)}

Wie schon angedeutet, behandelte dieses Werk nicht die Maschinenelemente im modernen Sinne, sondern die Grundlagen der Mechanik im Sinne der spätmittelalterlichen Statik und Dynamik. ${ }^{46}$ Uccelli rekonstruierte das Werk aus einer Fülle von Textauszügen der verschiedensten Leonardo-Handschriften, kannte aber nur einen direkten Hinweis, den auf ein viertes Buch dieses Titels im Codex Atlanticus fol. 161r (58r-a). Uccellis vier Kapitel oder Abschnitte mit Bezug auf das verlorene Werk behandeln einfache Zug- und Hebevorrichtungen („De' semplici tirari e alzari“), Flaschenzüge (,Delle taglie“), Welle und Rad (,De polo et rota“) sowie die Schraube („Della vite“). ${ }^{47}$ Es ist keineswegs sicher, ob diese Inhalte die Gesamtkonzeption von Leonardos Elementi macchinali ausreichend beschreiben. Tatsächlich behandelte der Text außer den konkreten Maschinen und Maschinenelementen auch die grundlegenden theoretischen Probleme der zeitgenössischen Statik und Dynamik.

Paolo Galluzzi, der verdiente Leiter des Instituts für die Geschichte der Naturwissenschaften in Florenz (jetzt Istituto Galilei), bespricht im vierten Band der großen Storia della Scienza an prominenter Stelle das verlorene Werk 1996 und erneut 2001. Für ihn steht ein enger Zusammenhang mit dem Inhalt des Codex Madrid I außer Frage. Er behandelt die Mechanik Leonardos deshalb ausgehend von einer näheren Charakterisierung der Elementi macchinali. Bekannt sind ihm ,vielfältige direkte Verweise“, von denen er drei besonders aufschlussreiche „riferimenti“ aus dem Codex Atlanticus hervorhebt (fol. 444r von etwa 1500, fol. $161 \mathrm{r}$ von ca. 1504 und fol 220v, vgl. weiter unten). Insgesamt habe das Werk wie der Codex Madrid I zwei Hauptteile gehabt, einen theoretischen und einen praktischen. Der Titel sei in Analogie zu den geometrischen Elementen des Euklid gewählt, besonders zahlreich seien die Verweise auf das vierte Buch. ${ }^{48}$

$46 \mathrm{Zu}$ diesen modernen Begriffen in ihrer Anwendung auf die mittelalterliche Mechanik vgl. die Artikel im Lexikon des Mittelalters: Edward Grant, Dynamik, Bd. 3, Sp. 1493-97, W. Knorr, Statik, Bd. 8, Sp. 64-66, E. Sylla, Kinematik, Bd. 5, Sp. 1156-58.

47 Uccelli (wie Anm. 6), S. 465-552. Eine übersichtliche und verständliche Einführung in die neuere Lehre bietet Friedrich Barth, Die Maschinenelemente, Berlin, Leipzig 1915.

48 Paolo Galluzzi, Gli ingegneri del Rinascimento da Brunelleschi a Leonardo da Vinci, Florenz 1996, S. 73f.; erweiterte Fassung in: Storia della Scienza 4, Rom 2001, S. 942 982, bes. 975 . 
Als direkte Verweise sind uns jetzt 16 Stellen bekannt. Wir ordnen sie nach Möglichkeit in der Abfolge des verlorenen Werkes. Zur Übersetzung der nur italienisch zitierten Stellen vgl. die Ausgabe von Reti (wie Amm. 7).

$\begin{array}{ll}\text { E.M. } & \text { Verweis } \\ \text { Buch über } & \text { Madrid I } \\ \text { Stützen } & 171 \mathrm{r}-\mathrm{a} \\ \text { (sostenta- } & \\ \text { coli) } & \end{array}$

Atl. 220v

1

2,5

2,9

3,5

Volo 8v

Atl. $185 \mathrm{v}$

\section{Text}

Hinweis auf Beginn eines künftigen Buches über Stützen „Principio del libro“. Inc.: „Quella parte del sostentaculo“.

„Elementi machinali. Del peso proporzionato alla potenzia che 'l move s'ha a considerare della resistenza del mezzo dove tal peso è mosso, e di questo si farà un trattato".

„Provasi per la prima delli Elementi machinali che nostra come le cose poste in bilancia, le quali sien percosse di là dal centro della lor gravitá, mandano in basso le parte opposite poste di qua dal predetto centro".

\section{Kommentar}

Gleiche Angabe „Principio del libro" mit gleichem Incipit im Atlanticus fol. 541v (202v-b)

Die Bemerkung gehört noch in die Planungsphase des angekündigten Traktates.

Die Hs. Turin datiert von ca. 1505 .
„Adunque a ha $3 / 8$ e b n'ha $5 / 8$.
Passt nicht zu Euklid Ora per la quinta del secondo delli 2,5 ; daher eher zu den Elementi, a cubo è in potenzia Elementi macchinali di discendere in $\mathbf{d}$ pel peso suo (Statik). naturale che è una libbra, ed è in potenzia di discendere in e per $3 / 4$ d'essa libbra“.
9 Paris ms. I „Per la nona del secondo degli Hs. von 1497-1499. Elementi che dice: il centro d'ogni Kein Bezug zu Euklid gravità sospesa si ferma sotto $\quad 2,9$. Somit wohl frü- il centro del suo sostentaculo“. her Beleg für die Ele- menti macchinali.
„Imperò che s'elli è 4 in $\mathbf{n}$, e' Gemeint sind der Fla- non ne pon 4 in $\mathbf{b}$, perché le 2 schenzug und die Be- carrucole n'han consumato parte, lastung der Seile (Ma- come si mostra nella quinta del drid I, fol. $154 \mathrm{v}-155 \mathrm{r}$ terzo degli Elementi machinali; und öfter).




\section{4,5}

Atl. $161 \mathrm{r}$

Volo $12 \mathrm{v}$

Atl. $444 \mathrm{r}$

Anat. $147 \mathrm{r}$ onde per questo e per quel ch'è

detto, la corda $\mathbf{n}$ sente molto più

fatica che la corda $\mathbf{c}^{\text {“. }}$

„De’ due cubi i quali son doppi uno all'altro, come si prova nel quarto delli Elementi Macchinali da me composto“.

„Oltre a di queso se 1'uccelo vorrà cadere colla testa in giù con parte d'obliquità che lo porti riverscio, questo non potrà accadere, perché la parte più lieve sarebbe sotto alla più grave e verrebbe a discender prima il lieve che ' 1 grave, la qual cosa in lungo discenso è imp[o]scibile, come si prova nel quarto de li Elementi machinali“".

„Se fai con carri, essi non possano fare 'n una medesima altezza più corta via che per obbliquità mezzana, [colla] quale a ogni corpo si rende la metà della sua gravità naturale, come provai nella quinta decima conclusione del quarto libro delli Elementi machinali da me composto. Nel medesimo tirare obblico ogni motore diminuisce la metà di sua potenzia, come in detto libro è provato".

„Dico la natura aver fatto tali nervi, ovver corde, appiccati alla stremi delli sproni di tali spondili del collo, perché, se la corda 'é posta in $\mathbf{m} \mathbf{n}$, essa volta più facilmente tale spondile che s'ella fussi posta in b a; perché $\mathbf{m} \mathbf{n}$ è appiccata a più lunga lieva che $\mathbf{b} \mathbf{a}$, ed ha tanto più potenzia quanto essa è più lunga, com'è
Beleg für Leonardos Autorschaft (Uccelli [wie Anm. 6], S. 465).

Entstehung der Maschinenelemente vor dem Buch über den Vogelflug (dt. Übersetzung Schneider [wie Anm. 14], S. 68.

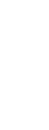

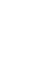

Weiterer Beleg für

Leonardos Autor-

schaft (nach Galluzzi von ca. 1500). Das

vierte Buch behandelte offenbar die Zugkräfte und enthielt „conclusiones“.

Übertragung mechanischer Prinzipien aus den Elementi macchiauf die Anatomie des Menschen 
provato nella quinta del quarto

delli Elementi macchinali“.

22,13 Atl. 10r „Le quali potenzie son vere, com'è provato nella tredicesima del ventiduesimo delli Elementi Macchinali da me composti““.

Anat. 143r „Fa' che '1 libro delli Elementi macchinali colla sua pratica vada innanzi alla dimostrazione del moto e forza dell'omo e altri animali, e mediante quelli tu potrai provare ogni tua proposizione“.

Arundel Elementi machinali- Über195v legungen zum Kräfteverhältnis (P55r) in geometrischer Darstellung; Dreiecke.

Sehr große Lücke zwischen dem 4. und 22. Teil des Werkes.

Das Buch über die Maschinenelemente soll (nach 1500) an die Spitze einer Studie über den Bewegungsablauf des Menschen treten.

Datiert auf 1500 bis 1505. (Texte bei Uccelli [wie Anm. 6], S. $343,353,474,529 f$.)

Arundel Elementi machinali-Studien

147r (P 76) zu Waage und Gewicht.

Arundel Elementi machinali-Kräfte6v (P 120v) verhältnisse

Arundel Elementi machinali-Kräfte-

7r (P 121r) verhältnisse

Paris E „E dato la potenzia del motore fol. $2 \mathrm{r}$ e la grossezza della vite e lunghezza della lieva, si ricerca il peso del mobile. Ora ricerca nel libro delli Elementi macchinali e quivi troverrai la definizione della vite e ciò che qui di sopra si dimanda“.
Uccelli (wie Anm. 6) S. 157 u. $178 f$.

Uccelli (wie Anm. 6) S. 469f., 181 u. 193. Ms. Paris E stammt aus den Jahren 1513 bis 1515 und behandelt vornehmlich statische Probleme (scientia de ponderibus"), hier Schraube und Hebel.

In drei von 16 Fällen ist, wie man sieht, die Autorschaft Leonardos ausdrücklich vermerkt („,da me composto“). Vor allem beim vierten Buch dominieren die Verweise auf Beweisführungen („come si prova, come provai“ etc.), die das Werk offenbar auch enthielt. Inhaltliche Schwerpunkte waren sowohl statische wie dynamische Aspekte der Mechanik, d.h. Gleichgewicht, Schwere, Bewegung, Gewicht, Kraft, Theorie des Rades und des Schwungrades, am Schluss aber auch Maschinen, wie aus einem frühen Plan Leonardos 
für den Aufbau dieses Werkes hervorgeht. Er notierte auf einem Blatt des Codex Atlanticus: ${ }^{49}$

Parla prima del moto, poi del peso, perché nasce dal moto, poi della forza che nasce dal peso e moto, po' della percussione che nasce da peso, moto e spesso dalla forza. ${ }^{50}$

Di' del moto e impeto. ${ }^{51}$

Di' delle rote che voltano per un sol verso.

Dirai delle rote che vanno e tornano.

Di' della rota dell'aumento.

Di' delle viti perpetue.

Di' delle lieve in prima e bilance.

Di' de' mulini e altri stromenti da movere e da gittare.

Di' [de'] denti.

Dirai prima de' poli e di chi li riceve a minuto o, po' delle rote e carelli sanza denti, poi delle rote e carello con corda e poi con denti, e nature e moto e regola, e dirai quali denti sono più laldati.
Sprich erst über Bewegung, dann über Gewicht, denn es entsteht aus der Bewegung, dann von der Kraft, die aus Gewicht und Bewegung entsteht, dann vom Stoß, der aus Gewicht, Bewegung und oft Krafteinsatz entsteht.

Sprich von Bewegung und Impetus. Sprich von Rädern, die sich nur in eine Richtung drehen.

Sprich von Rädern, die sich hin- und zurückdrehen.

Sprich vom Schwungrad.

Sprich von Endlosschrauben.

Sprich erst von den Hebeln und [dann von?] Waagen.

Sprich von Mühlen und anderen Antriebs- und Wurfmaschinen.

Sprich [von Zahnrädern und] Zähnen. Sprich erst von Wellen und ihren Lagern, dann von Rädern und Rädchen ohne Zähne, dann von Rädern und Rädchen mit Seil, dann mit Zähnen, [nach] Arten, Bewegung und Regel [?], und dann sage ich, welche Zähne besonders zu empfehlen sind.

Wie in der Tabelle zur Sammlung Windsor, Anatomie fol. 143r, bereits bemerkt, muss das Buch über die Maschinenelemente bereits vorhanden gewesen sein, als Leonardo den Satz schrieb: „Mach, dass das Buch über die Maschinenelemente mit seiner Praxis an den Anfang der Darstellung der Bewegung und Kraft von Mensch und Tieren trete, damit kannst du jede deiner

49 Codex Atlanticus, fol. 421v, alt 155v-b; vgl. Marinoni/Pedretti (wie Anm. 13), S. 778.

50 Vgl. Uccelli (wie Anm. 6), S. XXI, der die Stelle vergleicht mit Aussagen im Atlanticus, fol. 324r (alt 117r.c) und einem Fragment in Venedig (Ed. Uzielli 1884): Im Atlanticus heißt es: „tratta prima de' pesi e poi de' sua sostentacoli, e poi della sua confregazione e poi del moto suo e in ultimo della sua percussione.“ („Behandle zuerst die Lasten, dann ihre Stützen, dann ihre Reibung und Bewegung, zuletzt den Stoß“; vgl. auch Uccelli (wie Anm. 20), S. 440-449.

51 Im Sinne von ,parla ‘ fasse ich ,di‘ als Imperativ auf, bei Anpassung an ,dirai ‘ ergibt sich inhaltlich kein wesentlicher Unterschied. Zu Drehling mit Seil bzw. Riemen vgl. Madrid I, fol. $30 \mathrm{v}-31 \mathrm{r}$. 
Aussagen beweisen“. Dasselbe gilt für den Zeitpunkt der Anlage der Turiner Handschrift über den Vogelflug (ca. 1505), dort soll das Buch über die Maschinenelemente an die Spitze einer Studie über den Bewegungsablauf des Menschen treten. Es gilt aber auch schon 1497 bis 1499 in der Handschrift Paris I, wo es um die Position der Stütze geht (sostentaculo). Diese Handschrift bietet damit den bisher frühesten Beleg. Im Codex Madrid I dagegen wird das Buch über Maschinenelemente als Titel noch nicht zitiert. ${ }^{52}$ Als Entstehungszeit für die Niederschrift der systematisierten Überarbeitung von Madrid I sind damit die Jahre 1495 bis 1499 anzunehmen. Ob das Werk Leonardo 1513 bis 1515 bei der Niederschrift von Ms. E (letzter Verweis) noch vorlag, erscheint dagegen unsicher, da nicht klar ist, an wen sich die Aufforderung richtet, in ihm die Definition der Schraube (vite) zu suchen. Spricht Leonardo zu einem Leser oder zu sich selbst? In dieser Zeit war er viel auf Reisen und könnte das gesuchte Manuskript nicht zur Hand gehabt haben.

Den frühesten Ausblick auf die künftigen Elementi macchinali eröffnet Leonardo wahrscheinlich schon im Codex Madrid I fol. 82r. Diese dichte Zusammenstellung diverser Themen ist ganz auf die Zukunft gerichtet. Sie beginnt mit Überlegungen zur Schraube im einzelnen. Im zweiten Abschnitt weitet sich der Blick, die zu behandelnde Vielfalt der Themen wird immer größer:

„Dann wird mittels Linien über die Zurüstungen [,armadure“] selbst zu reden sein, dann über die Hebel als solche, dann über die Stärke der Träger und über ihre Dauerhaftigkeit und ihre Stützen [,sostentacoli'], ferner über den Unterschied zwischen dem Hebel mit kontinuierlicher Belastung während seiner Arbeit [...]. Es wird sodann über das Sperrrad und seine Klinke gesprochen, über das Schwungrad und den Schwung der Bewegung, über die Beschaffenheit der Achsen und ihre Seile, Flaschenzüge, Winden und Walzen. Es geht auch darum, Luft unter Wasser zu bringen und sehr große Gewichte an ihm hochzuziehen".

Fast alle Themen mit Ausnahme des letzten erscheinen zwar auch schon im Codex Madrid I selbst, aber nicht in dieser Reihenfolge. Das achtfach benutzte Futurum der Verbformen im italienischen Urtext zeigt eindeutig, dass ein künftiges Werk gemeint ist, welches die Dinge in einer klar konzipierten Abfolge behandeln sollte.

Ein weiterer Hinweis auf das künftige Werk im Codex Madrid I befindet sich auf fol. 171r. Hier markiert Leonardo den Satz „Quella parte del sostenta-

52 Paola Manni, Percorsi nella lingua di Leonardo: grafie, forme, parole, XLVIII Lettura Vinciana, Florenz 2008, S. 18 notiert zum Codex Madrid I: „ci restituisce con ogni probabilità la fase più avanzata del progettato libro sugli ,elementi macchinali““. Das möglicherweise nicht sehr umfangreiche Werk muss aber nicht nur geplant gewesen sein, sondern wirklich existiert haben. 
culo sarà più debole che dal suo fermamento è più lontana“ („,Der Teil der Stütze ist schwächer, der von ihrer Befestigung weiter entfernt ist") durch einen seitlichen Bogen, ein Verfahren, das er nach Reti oft anwendet, um eine Übernahme in eine andere Handschrift oder in ein anderes Werk vorzubereiten. Auch in Madrid I finden sich zahlreiche Beispiele für diese Vorgehensweise, oft deutet ein feiner Querstrich durch den Bogen an, dass die Übernahme erfolgt ist. Die Stellen sind in der Edition Retis angezeigt, sie häufen sich in den Abschnitten zu Stoß und Kraft, Gewicht, Schlag, Bewegung. Auf fol. 191r bis 178r, dem rückläufig zu lesenden Anfang des theoretischen Teils, sind fast alle Kurzdefinitionen in dieser Weise markiert. Wesentlich ist auf fol. 171r, dass über dem Satz noch eine Angabe nachgetragen ist. Sie verweist in aller Deutlichkeit auf den Anfang eines künftigen Buches über Stützen: ,principio del libro“. Die Tabelle der erhaltenen Verweise auf die Elementi macchinali reicht nicht aus, um die Position dieses Buches innerhalb des Werkes zu bestimmen. Der Begriff ,sostentacoli‘ in 2, 9 eröffnet aber eine Option auf einen wichtigen Teil, der in der Planung des Codex Atlanticus fol. 421v noch nicht oder nur mit der Bezeichnung ,peso' genannt ist. Gewichte benötigen auch Stützen. Auffällig ferner, dass Leonardo den Satz, der unter der Angabe ,principio del libro' steht, nachträglich mehrfach geändert hat.

Hinweise auf Buchanfänge im Werk Leonardos kennen wir auch aus anderen Handschriften. Wichtig ist vor allem Codex Atlanticus fol. 542r, denn hier handelt es sich um die gleiche inhaltliche Aussage: „Quella parte del sostentaculo sarà più debole che dal suo fermamento è più lontana"“. ${ }^{53}$ Zum Thema Stützen, einem zentralen Teil der Statik, hat Leonardo viel geschrieben, so schon in seinem frühen Skizzenbuch Paris A fol. 46r, wo an zwei Stellen von der Schwäche einer Stütze die Rede ist. Wie wir bereits sahen, wollte er wesentlich später im Ms. Paris E erneut auf das Thema Stützen zurückkommen.

Zur Klärung seien im Weiteren wichtige Einzelaussagen über das künftige Werk besprochen. Pedretti verweist auf inhaltliche Überschneidungen der Elementi macchinali mit dem zweiten Teil von Madrid I. ${ }^{54}$ Für eine direkte Identifikation mit dieser Handschrift reichen sie nicht aus. Madrid I liegt vor dem verlorenen Werk und ist ein noch unsystematischer Versuch einer möglichst umfassenden Behandlung von Theorie und Praxis der Mechanik, somit eine Vorarbeit auf breiter Basis, nicht das Werk selbst.

53 Die weiteren Stellen sind: 1. Ms. Paris F, fol. 66v (a. 1508): „Principio del libro“, offenbar Anfang eines Buches über die Bildung von Wassertropfen; vgl. Madrid I, fol. 1r oben (Nachtrag?) und Schneider (wie Anm. 16), S. 29-33 (Tropfen). 2. Ms. Paris F, fol. 95r: „Non si può difinire qui per carestia di carta, ma in verso il principio del libro a carta 40 , che lì è difinita" (fol. 40 war offenbar der Beginn eines Buches über die Intensität von Lichtstrahlen). 3. Ms. Paris I, fol. 171 r (a. 1497-1499): „Principio del libro dell'acque“ (zu diesem Buch vgl. oben Abschnitt 2).

54 Marinoni/Pedretti (wie Anm. 13), Bd. 2, S. 777. 
Ladislao Reti bemerkt zu dem Werk über „Maschinenelemente“, es werde in den Schriften Leonardos oft erwähnt, und es sei ein Werk in vier Bänden gewesen. „Wir wissen jetzt, daß er nicht im Codex Madrid I, sondern in einem anderen, in vier Bücher oder Bände eingeteilten Werk die Theorie der mechanischen Bewegung behandelt hat.“ Er fährt fort: „Auf einer Seite des Codex Madrid I (fol. 96v) sagt Leonardo: ,Wenn der Apparat einmal geschaffen ist, erfordert die Notwendigkeit seines Funktionierens die Form seiner Glieder. Diese sind von unbegrenzter Vielfalt, aber alle müssen den Regeln der vier Bände unterworfen sein" ". 55

Die zitierte Stelle mit Bezug auf vier Bände befindet sich, ohne inhaltlichen Zusammenhang, über Zeichnungen von Türscharnieren für Pavillons im Schloss von Vigevano. Im Originaltext lautet sie: „Creato che ffia lo sstrumento, la neciessità della sua operatione dimanda la forma delle sue menbra. Le quale son d'infinite figure, ma pure tutte fien sottoposste a queste regole de' 4 volumi“. Inhaltlich scheint dies zu den Grundlagen des Maschinenbaus zu passen. Die vier Bände wären aber nicht selbstverständlich, wenn es, wie in der Tabelle angezeigt, tatsächlich ein 22. Buch dieser Maschinenelemente gegeben hat (die Zahl ventiduesimo ist autograph und voll ausgeschrieben). Die Frage ist also: Waren die mindestens 22 Bücher der Elementi macchinali zusammengefasst in den genannten vier Bänden? Dies erscheint zunächst als einfachste Möglichkeit, doch der Bezug auf die Elementi macchinali würde nach sich ziehen, dass der Satz am oberen Rand von fol. 96v im Codex Madrid I ein direkter Hinweis auf das spätere Werk wäre. Bei näherer Betrachtung erscheint das als unwahrscheinlich, denn die beiden letzten Zeilen des Eintrags sind aus Raumgründen deutlich zusammengepresst und somit nachgetragen. ${ }^{56}$ Ausgeschlossen erscheint ein Bezug der vier Bände auf ein wichtiges Werk in vier Büchern zur Statik aus dem 13. Jahrhundert (De ratione ponderis), zugeschrieben dem häufig erwähnten Mathematiker Jordanus de Nemore. Leonardo hat diese Schrift zwar gut gekannt und oft benutzt, aber Bände sind nicht dasselbe wie Bücher. ${ }^{57}$

Eine Seite im Codex Atlanticus beschäftigt sich mit den Bauarbeiten an einem Kanal für Cesare Borgia und kann nach Reti auf 1502 datiert werden. Hier erwähnt Leonardo ,die 15. Schlussfolgerung in meinem 4. Buch über die Elemente der Maschinen“ (Atlanticus, fol. 444v, alt 164a). Dieser Hinweis ist eindeutig, doch auch hier ist Buch nicht Band. Zunehmend problematisch wird es, wenn Reti hinzufügt: „Offensichtlich wird auf diese Bücher auf zahlreichen Seiten des Codex Madrid I hingewiesen, zum Nachweis

55 Reti (wie Anm. 25), S. 275.

56 Über fol. 70r findet sich der formal einmalige Titel: „Della mutatione de' siti libro 3“. Ein Zusammenhang zu den auf fol. 96v genannten „4 volumi“ ist auch hier ausgeschlossen.

57 Erstausgabe als „Jordani opusculum de ponderositate“ durch Nicolò Tartaglia, Venedig 1565. Kritische Ausgabe mit Kommentar bei Moody/Clagett (wie Anm. 3), S. 167-227 u. S. 388-412 (Kommentar). 
mechanischer Demonstrationen und mit Zahlen, die ähnliche Unterteilungen bezeichnen: entsprechend dem 7. [Abschnitt] des 4. [Kapitels] über Kraft und Widerstand“. Reti ergänzt zwar mit Recht: „Da der Codex Madrid I nicht so eingeteilt ist, kann er nicht mit dem Werk Elementi macchinali identifiziert werden, das man leider für verloren erklären muss". Er fügt aber weiter hinzu und gerät damit wieder ins Hypothetische: „Das eigentliche Buch über Maschinenelemente ist der erste Abschnitt des Codex Madrid I, der sich mit angewandter Mechanik befasst. Der zweite Teil über die Theorie könnte mit den vier verlorenen Büchern Ähnlichkeit haben, ist aber nicht das Original" ${ }^{58}$

Schwierig zu beurteilen sind außerdem die in der Tabelle gegen Ende angezeigten vier umfangreichen Abschnitte aus dem Codex Arundel. Hier fehlen die Hinweise auf Ober- und Unterabschnitte, um sie eindeutig dem Werk über Elementi macchinali zuzurechnen. Die inhaltliche Zugehörigkeit unterliegt aber keinem Zweifel. Pedretti und Vecce ordnen diese Abschnitte verschiedenen Zeitabschnitten der Jahre 1500 bis 1508 zu. Die Zugehörigkeit der „bifogli“ im Codex Arundel zu bestimmten Lagen konnte noch nicht geklärt werden. Bei einer künftigen Rekonstruktion der Elementi macchinali werden diese umfangreichen Abschnitte trotzdem eine wichtige Rolle spielen. ${ }^{59}$

Als letzte hat die amerikanische Romanistin Marina della Putta Johnston in ihrer Dissertation von 2000 versucht, eine Identität zwischen dem Werk über Maschinenelemente und dem Codex Madrid I herzustellen. Ihr Bemühen führte zu keinem klaren Ergebnis, Augusto Marinoni ließ sich nicht überzeugen. ${ }^{60}$

\section{Kurzverweise auf ein neunteiliges Werk vor Codex Madrid I}

Dieselbe Autorin beschäftigte sich in ähnlicher Weise mit Kurzverweisen des Codex Madrid I, die jeweils rein numerisch auf einen Ober- und Unterabschnitt verweisen: „regola 3 a del $1^{\circ}$, 4 a del $1^{\circ}$, 5a del secondo“ etc. Sie

58 Ladislao Reti, Elemente der Maschinen, in: ders. (wie Anm. 25), S. 275 = Leonardo, der Erfinder, Darmstadt 1981, S. 151.

59 Leonardo da Vinci: Il Codice Arundel 263 nella British Library. Edizione in facsimile nel riordinamento cronologico dei suoi fascicoli, a cura di Carlo Pedretti. Trascrizione e note critiche a cura di Carlo Vecce, 2 Bde., Florenz 1998.

60 Johnston (wie Anm. 26), S. 49f. bezieht den folgenden Satz auf die Elementi macchinali, er meint aber die Luft und somit die allgemeine Elementenlehre: „Two notes of the Codex Atlanticus, fol. 185r and 870r (c. 1505), deal with the flight of birds [...]. In the second folio, Leonardo states that [...] given that the heaviest part of a bird is the center of its body, not the wings or the head, it is ,contro alla quarta del secondo degli Elementi, dove fu provato che ogni corpo che liberamente infra l'aria discende, discenderà in tal modo che la parte più grave si farà guida del moto““. Sie schreibt weiter: „Folio $185 \mathrm{r}$ contains a similar reference to a ,quarta di questo', that is, the same book of Elementi. Augusto Marinoni tells us that the referred work cannot be identified with similarly titled books from the time, hence he suggests that this may be an original work on mechanics by Leonardo, Elementi macchinali, which I believe to be Madrid I.“ 
betrachtete diese Angaben als interne Verweise innerhalb des Codex Madrid I und sah darin das stärkste Argument für die Einheit des Codex, obwohl der Band zwei Blattzählungen aufweist, die eine vorn beginnend fol. 1-95, die andere hinten jeweils auf den Versoseiten fol. 190v-96r:

"What provides the strongest connections, the unbreakable if flexible joints, between the components of the manuscript, is the system of internal references which underlines its material and conceptual unity [...]. Unlike in other manuscripts, in Codex Madrid I it is generally possible to follow the reference to its origin [...]. I propose that the first number in these references generally directs the reader to a page, or to a folio, in the quire indicated by the second number. Still the identification of the original passages is often highly problematic". ${ }^{61}$

Diese Deutung der Verweise als ,internal references“ innerhalb des Codex Madrid I ist in der Tat problematisch. Nur in einem einzigen Fall könnte sie annähernd zuzutreffen. Eine alte Lagenzählung, auf die Leonardo sich hätte beziehen können, fehlt jedoch. Somit fehlt schon die elementarste Voraussetzung für eine praktikable Handhabung des vorgeschlagenen Verfahrens. Die Blattzählungen im Codex Madrid I weisen zudem eher auf ein nachträgliches Zusammenbinden von zwei ursprünglich getrennten Arbeitsmanuskripten bzw. Werken. ${ }^{62}$ Letzthin muss der Versuch von Johnston, die über 40 numerischen Verweise auf die Lagen und Blätter des Codex Madrid I zu beziehen, als gescheitert angesehen werden. Viel eher verweisen sie auf ein früheres, bereits vorhandenes Werk.

Im Index seines Einleitungsbandes zur Edition von 1974 hatte Reti bereits 31 solche Kurzverweise angezeigt. Wir stellen hier 45 von ihnen im jeweiligen Zusammenhang dar und gliedern sie in der Abfolge der genannten Oberund Unterabschnitte. Zu deuten sind sie, wie schon angesprochen, als Teile eines verlorenen Werkes, das älter sein muss als Madrid I.

Die bisherige deutsche Übersetzung des Codex Madrid I spricht von Büchern und Kapiteln und löst alle Verweise in entsprechender Weise auf: „nach dem 5. Kapitel des siebten Buches“ statt „per la 5a del 7“ (nach der 5. des 7.). Für die Oberabschnitte mit immer männlich deklinierter Ziffer wird die Deutung als Buch durch Stellen wie „per la 5a del 7“, „de moto e fforza“ gestützt; mit „de moto e fforza“ ist eindeutig ein Titel gemeint; fragt sich nur,

61 Johnston (wie Anm. 26), S. 47; vgl. ebd. die Übersicht, S. 358 und ohne Einschränkung S. 24. In nur einem Fall (fol. 130r - „5a del 9“) trifft die vorgeschlagene Lösung annähernd zu. Nützlich erscheint der Hinweis auf die bisher unbemerkten ,preparatory drawings“ (S. 51-53).

62 Marinoni (wie Anm. 31), S. 59, vermutet mit guten Gründen, dass „Leonardo die beiden Bände schon unterschied, sie jedoch als zwei Teile ein und desselben Werkes angesehen hat". 


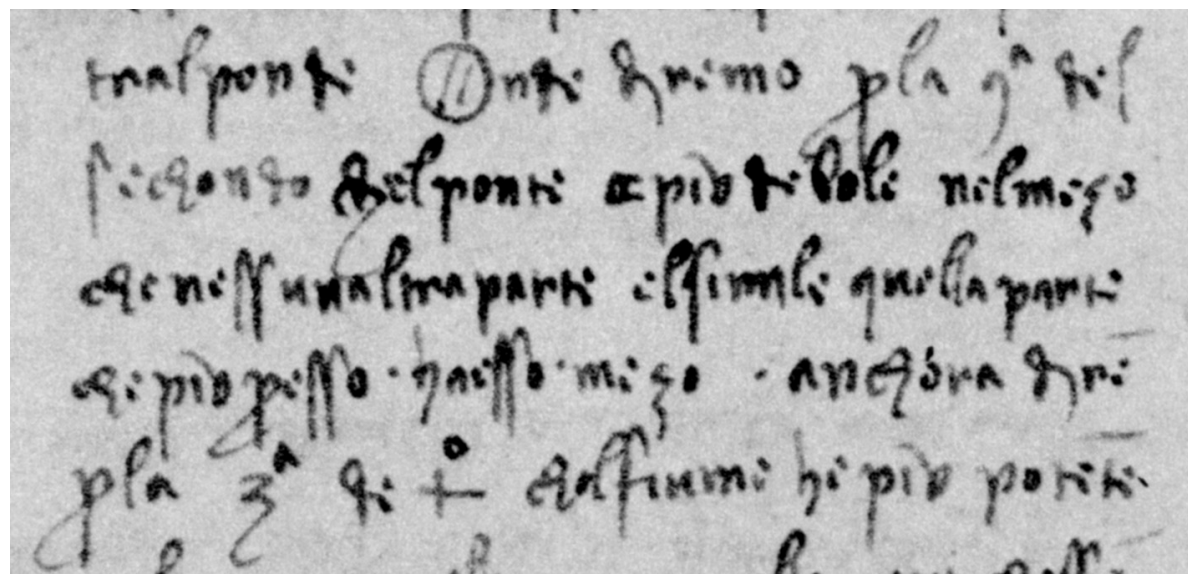

Abb. 3: Madrid I, fol. 144r (gespiegelt): „Onde diremo, per la 9a del secondo, che '1 ponte è più debole nel mezo che['n] nessun' altra parte e 'l simile quella parte ch' è più presso a esso mezo. Ancora diren, per la 3a de' $4[\ldots]^{\prime \prime}$.

ob 7 auf einen Teil dieses Buches als Ganzen weist oder das 7. Buch den Titel de moto e forza trug. ${ }^{63}$ Für die erste, immer weibliche Zahl ist Kapitel („,capitolo“) unwahrscheinlich. Diese erste Zahl verweist eher auf Unterabschnitte in Form von Sentenzen oder Regeln.

63 Vgl. in der Tabelle 2.28: ,per la 28a cunclusione del $2^{\circ}$ libro“. 


\section{Verweise im Codex Madrid I auf Ober- und Unterabschnitte eines neunteiligen Werkes (Teorica mechanica?) ${ }^{64}$}

\author{
Neuntei- Fol. Themen und Wortlaut \\ liges Werk
}

1. Hebel,

Kraft,

\section{Gewicht}

1,3

1,4

1,5
$94 \mathrm{r}$

\author{
De forza e peso (?)
} primo".
Inhaltliche

Entsprechung

in Madrid I

von hinten

(Auswahl)
$20 \mathrm{v}-26 \mathrm{r}$

(171r-165v)

$21 \mathrm{r}(170 \mathrm{v})$ fa essalieva sia h-f, e lla contralieva $\mathbf{f}-\mathbf{m}$.

E poi calcula colla regola della $3 \mathrm{a}$ del
1,6

(1)
73v Kräfteverhältnis. „Ora, e’ si trova in o 4 libre
di peso, e 'l bracio a-m è quadruplo al braccio
m-o. Onde, per la $4 \mathrm{a}$ del $1^{\circ}$, uno in b-a tirando
$(171 \mathrm{r}-165 \mathrm{v})$ sta equale con 4 di $\mathbf{o}^{\prime \prime}$.
114v Druck und Fall eines Gewichts. „Dico, che 25r? quanto più prieme, più forte prieme. E cquesto (166v) non nasscie per la sententia della 5a del primo, 169r (Balg) che dice, che 'l peso che più cade, più pesa, perchè ne' moti tardi non pò fare inpeto".
1,5 87r Statik eines Balkens, der an einem über Rol- 23v-25r len gelegten Seil hängt. „Dimandasi in che (168r-166v) grado d'obbliquità si fermerà il trave a-n.[...] m-c sostiene, per la 5 a del primo, tutto il trave“.
87r Mittelpunkt des Balkens unter Mittelpunkt seiner Aufhängung: ,overo che 'l cientro del trave venga, per la $6^{\circ}$, sotto il cientro del suo sostentaculo“.

$20 \mathrm{v}-26 \mathrm{r}$

64 Übersetzungen der nachfolgend zitierten Stellen enthält die Edition Retis von 1974 in mehreren modernen Sprachen. Ca. 20 weitere Kurzverweise der genannten Art fanden sich in den Codices Atlanticus, Forster I, 1 fol. 9v und 18r, Forster II, 2, Madrid II, Leicester und Paris E. Leonardo scheint das verlorene frühe Werk also auch um 1413 bis 1415 (Ms. E) noch benutzt zu haben. Insgesamt sind jetzt 45 Kurzverweise aus Madrid I und 23 aus anderen Handschriften bekannt. Die zusätzlichen Verweise lasse ich zunächst unberücksichtigt. 


$\begin{aligned} 1,7 \quad 127 \mathrm{v} & \text { Mittelpunkt der Schwere von Dreiecken. „E } 27 \mathrm{v} \\ & \text { per la 7a del primo, io trovo il triangolo a-b-d } \quad \text { (164r) } \\ & \text { e '1 triangolo b-c-d essere cole lor base sopra } \\ & \text { una medesima linia a-b-c, e ffinire 'n una } \\ & \text { medesima alteza, cioè in d“. }\end{aligned}$

\section{Gewichts- Gravità sospesa? verteilung}

65r Verteilung der Zugkräfte an einem schräg $28 \mathrm{r}-33 \mathrm{v}$ aufgehängten Gewicht. „Questo corpo sospeso, per la 5a del secondo, essendo 8 libre, 4 se $(163 \mathrm{v}-158 \mathrm{r})$ ne scarica in $\mathbf{f}$, e 4 in $\mathbf{r}^{\text {". }}$

2,5 $85 \mathrm{v} \quad$ Verteilung der Last auf die Arme einer Blattfeder oder eines Bogens. „Se '1 braccio c si fermassi, el braccio $\mathbf{d}$ moverebbe esse 200 libre 2 braccia, e per la $5^{\mathrm{a}}$ del $2^{\circ}$ fia inpossibile“.

122r Rad als Hebel: Bei gleicher Reibung, konzentriert auf die Achse, wirkt die Speiche als $163 \mathrm{v}$ Hebelarm. ,Vedi per la 5a del $2^{\circ}$, che 40 libre di confregatione non tornano altro che una libra di fatica a chi la move".

\section{2,5}

$141 \mathrm{r}$ Verteilung von Kippmomenten ungleich hoher 31v Quader. „E sse 11'uno (per la 5a del secondo) piglia la metà del suo quarto circulo, cioè la sua basa b-r, l'altro piglia il $4^{\circ}$ apunto“.

$2,9 \quad 63 \mathrm{v}$ Gewichtsminderung durch Anheben eines Gegenhebels (Ändern der Lastverteilung an den Auflagepunkten). ,Vera cosa è, che quanto il peso attacato in $\mathbf{X}$, quanto più s' alza, più si fa lieve, in modo che quando la punta della contralieva s' è alzata insino in $\mathbf{b}$, el peso è diminuito $1 / 10$, come si dimostra nel $9^{\circ}$ del $2^{\circ}$, per la linia $\mathbf{r}-\mathbf{S}$ e b-r, onde a sentirà 5 e $1 / 2$, e b $41 / 2$ “. 
2,20

2,20

$2,28^{\circ}$ cunclusione

2

penultima

2

penultima

3. Druckverteilung 19v Natürliches Streben nach Kongruenz von Gewichts- und Kraftlinien einer Schraube. „E dal mezo d'essa madre in giù la vite farà forza dall' oposita parte del peso, la qual forza non renderà manco faticoso il moto ch' ella si faciessi dal mezo in su. Questo si dimostra nella 20a del secondo, come il cientro d'ogni gravità desidera stare sotto il cientro del suo sostentaculo“"

97r Besserer Wirkungsgrad eines Tretrads mit $94 \mathrm{v}$ Außenantrieb bzw. längerem Krafthebel. „Ora, (97r) per 1la 20a del $2^{\circ}$, vedi che proportione ha dal polo della rota la distantia di d, colla distantia di $\mathbf{c}$, che $\mathbf{d}-\mathbf{b}$ è tripla a $\mathbf{b}-\mathbf{a}^{\prime \prime}$.

153v Verhältnis zweier Gewichte. „Quella proportione che ha la linia spirituale $\mathbf{m - d}$ colla linia reale $\mathbf{m}-\mathbf{c}$, tale arà il peso di $\mathbf{c}$ col peso di $\mathbf{S}$ per la 28 a cunclusione del $2^{\circ}$ libro“".

26r Berührung im Kugellager zwischen Gewicht 90r und Widerstand. „Ma lle palle, o voglian dire i curri, che saranno alquanto disstanti, non tocherano infra 'l peso e lla sua resistentia altro che in punto, come nella penultima del $2^{\circ}$ si dimostra“.

148v Belastung der Enden eines Seils. „quanto meno la [corda] piega, più li agrava li stremi, cioè il sostentaculo delli stremi, perchè della corda il peso è per tutto a u' modo, per la penultima del $2^{\circ}$ “.

\section{Dei pesi?}

102v Kraft einer Welle auf anderen Rädern. „Tutta la forza del polo $\mathbf{f}$ s' astende in moto traversale, over laterale, e ss' apogia sopra i poli p-q. I quali poli ricieverai sopra altre rote. E per la 5 a del $3^{\circ}$, farà bono ofitio“.

$114 \mathrm{v}$ Druckluft verteilt sich gleichmäßig im Behälter. „Le 100 libre del peso che prieme il mantice, fa che ll'aria spingie e vol fugire del vacuo 

d'esso mantace. E per la 5a del $3^{\circ}$, essa spin- gie equalmente in ogni parte di sua abitatione“.
3,8
3
penultima
121r Gleicher Druckwiderstand bei Reibung in einer Schraube. „Ho mostrato nella penultima del $3^{\circ}$, come tutte le varie quantità delle con- fregationi che pò fare un medesimo peso sopra una medesima natura di resistentia over sostentaculo, essere tutti d'equal fatica al suo motore".

\section{Kraft \\ und Widerstand \\ De potentia e resistentia

4,3

4,7

4,7: de potentia e resistentia

4 penultima

\section{Bewegung und Schwere}

5,3 teorica

144r Verhältnis der Kraft eines Flusses zum Widerstand einer Brücke. ,Ancora diren, per la $3 \mathrm{a}$ de' $4^{\circ}$, che 'l fiume è più potente in quelle parti che sson più presso al mezo che altrove, essendo di dirito corso. Onde il ponte fia dificile a regiere cola sua parte più debole alla parte più potente dell' acque“.

69v Größere Kraft am längeren Hebel. „Quanto il perpendiculare del peso che move questa rota fia più distante al cientro dello suo polo, tanto fia di magior potentia, per la 7 a del $4^{\circ}$.

97v Kraft und Widerstand im Getriebe. „Ma sse lla roca move tal rota, e che la corda del peso si racolga al suo albero, cierto la roca moverà facilmente essa rota e sua denti saran molto durabili. Per la 7 a del $4^{\circ}$, de potentia e resistentia“.

153v Die Senkrechte der Mitte eines Gewichtes stehe am Lastpunkt ihrer Basis. „,Ora, per la penultima del $4^{\circ}$ diremo che '1 perpendiculare del cientro del peso di tale aste sia dove essa aste carica la basa del suo sostentaculo h-p, come dire; 1'aste b carica in a“.

\section{Moto e peso ?}

18v Antriebsrad und Drehrichtung im Getriebe einer Endlosschnecke. „Qui in questo strumento la rota c è il primo moto. E lla 
rota picola, per la $3 \mathrm{a}$ del $5^{\circ}$ della teorica, è causa che lla vite a si volta per lo medesimo verso della rota $\mathbf{c}^{\text {“. }}$

5,3 74r Mittelpunkt der Schwere. „Per la 3a del 5 $5^{\circ}$ il punto $\mathbf{M}$ fia il mezo della gravità n-f, il quale è 8 “.

5,7 73v Verhältnis der Arme einer Waage. „Per la $7 \mathrm{a}$ del $5^{\circ}$ ora noi diremo, che ttale corpo sia 8 libre di peso, onde se la prima corda fia sola quella che ttira il peso per piegarlo, guarda che proportione ha il suo braccio della bilancia e-a, col braccio a-n“"

5 (cfr 73v) 71v Hebelverhältnisse beim Kippen eines schweren Blockes. „Il peso che hai a piegare sull' angolo $\mathbf{S}$ sie il corpo $\mathbf{t} \mathbf{f} \mathbf{v} \mathbf{S}$, ch' è 8 libre. El quale, come ssai, per la 5a, tucto è in $\mathbf{z}^{\text {“6 }}$

5

teorica

5

penultima

6. Erschwerte

Bewegung,

Reibung

6,2

6,5

6,5

$51 \mathrm{v}$ Mehr Kraft bei größerer Bewegung. „Quando la rota $\mathbf{d}$ dà una volta intera, la rota $\mathbf{c}$ ne dà 10 e lla rota $\mathbf{b}$ ne dà 100 e così la rota a ne dà 1000 . Onde per l'oposito, si scanbia la forza, come detto nella 2 a del sesto di sopra, che dov' è magiore il moto, lì è magiore forza". disscienso di $\mathbf{r}$ pervenendo in $\mathbf{x}$ ha riavuti 11/12 del suo natural peso, per la 5a della mia teorica“".

172v Reibung auf horizontaler Ebene viel schwerer als auf senkrechter Ebene.,,Perché chiaro si manifesta che lla confregatione fatta sopra la linia equidiaciente è molto più dificile che quella ch' è ffatta per linia perpendiculare, come nella penultima del $5^{\circ}$ si dimosstra“.

\section{De confregatione}

Erschwerte Bewegung durch Berührung im Kugellager. „Ma sse lle palle, overo i curri, si tocherano in e lor moti, sanza conparatione renderanno più dificile il moto che sse essi fussino sanza contingientia. Inperochè i lor tocamenti si confregano per contrari moti, come nel $5^{\circ}$ del $6^{\circ}$ si dimostra, e ssi vengano per tal confregatione a contradire i moti“".

$47 \mathrm{v}$ Erschwerte Bewegung in Schlossmechanismen. „Ma i moti di tali ranpini [Haken] non sono equali, nè in forza nè in moto, perchè molto più corta è la linia $\mathbf{a}-\mathbf{c}$ che lla linia $\mathbf{b}-\mathbf{a}$. E per la $5 \mathrm{a}$ del $6^{\circ}$ a-b più move il suo ranpino, e con più fatica“". 


\begin{abstract}
6,5 70r Erschwerter Anschlag im Schlagwerk für Uhren: Hammer mit 15-fachem Gewicht. „Il contatto fatto dalla stanga dove si ferma il manico del martello, intre ll'anello $\mathbf{m}$ che lla ricieve, ha 15 tanti più peso sopra di sè che non ha l'anello opposito $\mathbf{n}$, per la 5 a del $6^{\circ}$. E ffermisi tal martello sopra che parte si voglia sopra la stanga $\mathbf{n}-\mathbf{m}$, che ssenpre il motore a harà equale fatiche“".

6,5 118r Eigenschaften kurzer und langer Wellen, Einfluss von Verschmutzung in der Schmierung der Lager. „I poli carichi da equale peso e di pari grosseze, in varie quantità di contatto, son d'equal facilità ne' lor moti, come dimostrai nella 5 a del sesto, dove tratta di confregatione de' corpi gravi“.
\end{abstract}

\title{
7. Belastung, Centri di gravità ? \\ Träger, Stützen
}

7,5 127v Bestimmen der Schwerpunkte von Dreiecken einer Waage durch Wägeversuch. ,E per la 5 a del $7^{\circ}$, io arò il mezo del peso dell' uno e dell' altro triangolo, e metterà al cimento della bilancia“.

7,5 139v Belastung von Bögen: jedes Gewicht ist ganz sein Träger. „Perchè tanto peso apunto fia sopra de' sua piedi quanto è quello che sta sopra del suo mezo, per la 5 a de' $7^{\circ}$, che dice ch' ogni peso è tutto per tutto il suo sostentaculo e ttutto in ogni parte di quello“.

7,5 140v Verringertes Gewicht von angehobenen Stützen. „I sostentaculi teorica n-m ciasscun per sè è 6 pesi, che nella loro equidiacentia ciasscuna loro stremità pesava 3 . Ora essendosi tanto levati che 11 'hano diminuito il $3^{\circ}$ della equidiacenza loro, la fronte co' ss' è alzata ha diminuito il terzo del suo peso, per la $5 \mathrm{a}$ de $7^{\circ}$ della teorica, sichè ciasscuna fronte ressta due pesi, che fanno insieme giunti 4 pesi“.

7,60 43r Belastung eines Seils durch ein Gewicht. „Chiaro si dimosstra per la 60 a del $7^{\circ}$, che lla corda equidiacente sente men fatica del peso da llei mosso, che per qualunque linia esso peso fussi tirato. Inperochè la corda equidiacente $\mathbf{f}-\mathbf{m}$ solamente è affaticata dalla confregatione che ffa esso peso colla terra donde si move“.

\section{Waage? Bilancia?}

$8,6 \quad 74 \mathrm{r} \quad$ Drehpunkt der Waage. „E per la 6a del $8^{\circ}, \mathbf{n}$ sie il polo d'una bilancia, le braccia della quale sono le linie r-n e n-M“". 


\section{Gleich- \\ Gravità e equilibrio? \\ gewicht \\ Lastpunkt, \\ Schwerpunkt}

$9,5 \quad 4 \mathrm{v}$

Gleichgewicht (Instrument zur Messung der Kraft eines Schraubengetriebes). „Io ho creato a mme la maesstra, la quale fedelmente mi mostra i sua secreti, e mi fa intendere come ogni libra posta in $\mathbf{n}$, mi sta pari con [...] posta in $\mathbf{f}$. E lla ragione si è, perchè, come ti mostrai nel $5^{\circ}$ del nono“.

9,5 73v Schwerpunkt in der Mitte der Basis. „Dico adunque, per la 5a del $9^{\circ}$ : tutta la gravità di tale corpo risiede nel mezzo della sua basa".

(9),5 71v Hebelarm beim Kippen eines schweren Blockes. „Il peso che hai a piegare sull' angolo $\mathbf{S}$ sie il corpo t-f-v-S, ch' è 8 libre. El quale, come ssai, per la 5a, tucto è in $\mathbf{z}^{\prime \prime}$.

9,7 143v Berechnung eines Gewichts durch Verschieben des Schwerpunkts. ,Se volli sapere quanto peso quessto triangolo dia al suo contrapeso, toli il cie[ntro] della sua gravità nel punto $\mathbf{b} \mathrm{e}$ guarda quanto esso sporta fori di c e per la 7 a del $9^{\circ}$ troverai la verità di questo peso“.

9,7 73v Winkel der Welle einer Waage. „E per la 7 a pure del $9^{\circ}$, dico le sopradette linie fare braccia alla bilancia, in quella parte dove essa è più vicina all' angolo $\mathbf{m}$. Il quale angolo viene a essere polo di tal bilancia, e ll'oposito braccio fia la linia m-o“"

9,7

teorica

140v Verminderte Gewichtskraft eines Keils, der nicht im rechten Winkel wirkt. „Ora ci resta che la potentia del conio non adopera colle sue linie per angoli recti, insieme colla giuntura che fa colle sue linie de' 2 travi, e per la 7a del nono della teorica a esso conio manca il peso che per essa 7a si dimosstra, quando sarà considerato la diminutione dell'angolo acuto a $\mathbf{o} \mathrm{S}$, quanto li manca a andare all'angolo recto“.

9,8 138v Anheben eines Schwerpunktes erschwert Hebelwirkung. „Questo in ogni grado di moto osserva una medesima natura di peso, perchè la gravità $\mathbf{a}-\mathbf{b}$ quanto più è alzata in $\mathbf{b}$, più si fa lieve e lla lieva che lla move più si fa dificile, per la $8 \mathrm{a}$ del nono“. 
Die Oberabschnitte dieser Übersicht entsprechen in etwa den von Uccelli 1940 rekonstruierten Büchern II bis IX unter seinen Libri di meccanica, wenn man von deren Reihenfolge absieht. Sowohl die deutschen wie die italienischen Überschriften sind hypothetisch aus den Verweisstellen abgeleitet, um zu versuchen, den Verweisen jeweils einen „gemeinsamen Nenner“ zuzuweisen. Auffällig ist die Dominanz der statischen Themen.

Die oben in Abschnitt 2 besprochenen Verweise auf andere Werke Leonardos, insbesondere Di moto e colpo, Di moto e resistentia, De moto e fforza, Della mutatione de' siti, De stracinatione, De impeto sind hier nicht aufgeführt. Sie korrelieren inhaltlich noch stärker mit Themen der aristotelischen Bewegungslehre und ihrer Anwendung. Ihre Zugehörigkeit zu dem hier rekonstruierten Werk ist nicht ausgeschlossen, doch betrachtete Leonardo es in ihrem Fall als notwendig, einen besonderen Titel anzugeben, was er für die neun Teile nicht tat.

Vereinfacht sprechen wir künftig von einem neunteiligen Werk zur vornehmlich theoretischen Mechanik mit Schwerpunkt auf statischen Themen. Nur die Abschnitte 4 bis 6 gehören eher zur Bewegungslehre. ${ }^{65}$ Abschnitt 6 De potentia e resistentia bildet darüber hinaus den theoretischen Hintergrund für Leonardos praktisches Bemühen um optimierte Energienutzung, wie sie in seinen Kugellagern und den Studien zur Minderung der Reibung hervortritt und nicht zuletzt in seiner Erfindung des Freilaufs. ${ }^{66}$

Dieses Werk muss früher entstanden sein als Madrid I, da die Verweise dort keine zahlenmäßige Entsprechung finden bzw. man eine Entsprechung, von zufälligen Ausnahmen abgesehen, für die Masse der Verweise dort nicht finden kann. Als Leonardo am Codex Madrid I arbeitete, war dieses verlorene Werk folglich bereits abgeschlossen; „di sopra“ im Verweis auf 6, 2 ist scheinbar der einzige Binnenverweis in Madrid I, entspricht aber eher nur einer irrtümlich übernommenen Formulierung.

Die Autorschaft Leonardos ergibt sich eindeutig aus den Formulierungen in der ersten Person: „ho mostrato nella penultima del $3^{\circ}$. Ora per la penultima del $4^{\circ}$ diremo, come nella $8^{\text {a }}$ del $3^{\circ}$ dimostrai, come ti mostrai nel $5^{\circ}$ del nono" etc. Die hier gewählten Vergangenheitsformen sprechen deutlich für ein bereits abgeschlossenes Werk.

65 Uccelli (wie Anm. 6), S. XX geht davon aus, dass Leonardo wie schon die klassische Antike eine klare Scheidung von Statik und Dynamik nicht kannte: „perché allora, come nell'antichità classica, non si faceva distinzione tra dinamica e statica“. Duhem und Clagett sprechen aber sehr wohl von einer mittelalterlichen Statik, der „scientia de ponderibus“. Vgl. auch die Artikel Statik und Dynamik im Lexikon des Mittelalters (wie Anm. 46).

66 Vgl. dazu die Studie von Frank Hasters zum Freilauf (Essay zur kommentierten InternetEdition von Codex Madrid I; vgl. Anm. 10) und Retis klassische Untersuchung zur Reibung bei Leonardo: Leonardo on Bearings and Gears, in: Scientific American 224, 1971, S. $100-110$. 
Einen Titel hat dieses Werk noch nicht, da Leonardo sich seine Angabe in den meisten Fällen erspart. Es gibt aber, wie die Tabelle zeigt, insgesamt vier Ausnahmen: bei 3,5, bei 7,5 und bei 9,7. Diese vier Verweise passen inhaltlich in die Systematik. So scheint Leonardo hier den Obertitel seines Traktats zu verraten. Wie auch immer man diese Hypothese beurteilt, der Titel passt inhaltlich gut. Die neun bekannten Teile gehören alle zur älteren theoretischen Mechanik. Teorica meccanica könnte der Titel vollständig geheißen haben.

Zum Thema Statik bei Leonardo da Vinci wird sich erst nach Bearbeitung des theoretischen Teiles von Codex Madrid I mehr ergeben. Die grundlegenden Forschungen sind, wie schon angedeutet, die von Duhem und Clagett. ${ }^{67}$ Nur an einer Stelle scheint Leonardo direkt auf den für die Statik wichtigen Namen des Jordanus de Nemore verwiesen zu haben: auf einer Art Notizzettel im Rahmen der Windsor-Manuskripte. Der Name des Jordanus erscheint auch dort in keinem Zusammenhang zu den wissenschaftlichen Entwürfen Leonardos. Wenn Leonardo ihn zitiert, so geschieht das eher unter Hinweis auf die Scientia de ponderibus.

\section{Folgerungen zur relativen Stellung des Codex Madrid I}

Von den 120 eigenen Schriften, die Leonardo gegen Ende seines Lebens mit dem Bedauern ansprach, sie nicht vollendet zu haben, sind umfangreiche Fragmente in einer bisher nur schwer zu beziffernden Zahl erhalten. Es fehlt eine vollständige Übersicht über diese Titel und eine Zusammenstellung der Fragmente, die eine Zuweisung zu diesen Titeln erlauben. In Abschnitt 2 dieser Arbeit ist das Problem anhand der einschlägigen Verweise des Codex Madrid I nur vorläufig angesprochen. Noch nicht klar ist, ob es sich jeweils um selbstständige Werke oder eventuell doch um Teile eines größeren Gesamtwerkes handeln soll.

Auch auf die Benutzung antiker Autoren (Abschnitt 3) wurde nur zur Vollständigkeit hingewiesen, um eine Typologie der Verweise in ihrer Verschiedenheit abzubilden. Zudem ist Euklid erst ab 1495 für Leonardo wichtig geworden, Archimedes noch später. Am Schluss (Abschnitt 5) ging es um ein verlorenes Werk über Aspekte der spätmittelalterlichen bzw. vormodernen Statik, ein Werk, das mindestens neun Teile umfasste, sämtlich theoretischer Natur. Auf dieses frühe Werk verweist Codex Madrid I (ca. 1493-1495) allein 47-mal, verteilt auf den gesamten Codex (weitere Belege aus anderen Handschriften konnten noch nicht berücksichtigt werden). Das verlorene Werk, das sich aus diesen Stellen ergibt, liegt chronologisch vor 1493-1495. Es war allem Anschein nach in seiner Systematik bereits abgeschlossen, als Leonardo mit der Arbeit an der Handschrift Madrid I begann. Teile dieses

67 Duhem (wie Anm. 37); Marshall Clagett, The Science of Mechanics in the Middle Ages, Madison, Wisconsin 1959, Kapitel 2. 
Werkes oder Entwürfe wird man vielleicht in den frühen Pariser Skizzenbüchern, in Forster I,2 oder dem Codex Arundel nachweisen können. Als Titel schlagen wir Teorica meccanica im Anschluss an vier Verweise des Codex Madrid I vor.

Als weiteres verlorenes Werk kommt hinzu der seit langem bekannte Titel eines Werkes über Elementi macchinali (Abschnitt 4). Die jetzt 16 ausdrücklichen Verweise auf dieses Werk stammen aus deutlich späteren Handschriften Leonardos, nämlich aus der Turiner Handschrift über den Vogelflug (1505), aus dem Codex Madrid II (1503-1505), aus den Pariser Notizbüchern I (1497-1499), K (1503-1507) und E (1513-1515), aus offensichtlich späten Blättern zur Anatomie, aus dem Codex Arundel und dem Codex Atlanticus, wo eines der einschlägigen Blätter nach Reti auf 1502 zu datieren ist.

Den frühesten Beleg liefert das Pariser Manuskript I von 1497 bis 1499. Leonardo hat demnach seine Elementi macchinali, auf die er im Codex Madrid I (1493-95) noch nicht verweist, vor dem Ende seiner ersten Mailänder Zeit abschließen können. Eine Motivation zu dieser Umarbeitung lieferte ihm seine Auseinandersetzung mit den Elementi geometrici des Euklid und die schon erwähnte Begegnung mit dem Mathematiker Luca Pacioli. In den Pariser Skizzenheften M, I und K ist dieser Vorgang nachzuvollziehen. In seinen späteren Arbeiten, denen des 16. Jahrhunderts, verschiebt sich, wie zuletzt vor allem Domenico Laurenza mit Nachdruck betont hat, Leonardos Interesse von der Mechanik der Maschinen zur Mechanik des menschlichen Körpers. ${ }^{68}$ Dabei verweist er immer wieder auf seine Elementi macchinali, aber gelegentlich auch noch auf das frühe neunteilige Werk der Teorica, das er offenbar auch um 1515 noch zur Hand hatte.

Der Umfang der Elementi macchinali betrug mindestens 22 Bücher oder Abschnitte, wenn wir der Angabe von fol. 10r im Codex Atlanticus vertrauen. Das wird auch in anderer Hinsicht deutlich, denn zu diesem Werk liegt im Codex Atlanticus auf fol. 421v ein detaillierter Inhaltsentwurf vor, der etwa 20 Abschnitte erkennen lässt. Die Aufzählung beginnt mit theoretischen Themen wie Bewegung, Gewicht, Kraft, Stoß, Impetus und geht danach über zu praktischen Dingen wie Räder, Schwungräder, Hebel, Waagen, Mühlen, Wurfmaschinen, Zähne bzw. Zahnräder, Wellen und Lager, Reibungsräder, Seil- und Riementriebe, Getrieberegeln. Bei genauer Auszählung kommt man auf 20 Abschnitte oder Bücher. Aus ihnen sind bei der Ausführung des Werkes mindestens 22 Bücher geworden.

Vergleicht man dieses Programm mit den Inhalten des heutigen Codex Madrid I und beginnt dort mit dem theoretischen Teil, so finden sich sämtliche Themen der Statik in dem erhaltenen Codex wieder. Ähnliches gilt für den praktischen Teil, wo freilich die Reihenfolge in keiner Weise eingehal-

68 Domenico Laurenza, Leonardo Anatomie, Stuttgart 2009. 
ten ist. Was dagegen in Madrid I fehlt, ist vor allem die systematische Zählung nach Ober- und Unterabschnitten. Auf sie verweist Leonardo bei der Erwähnung seiner Elementi macchinali in neun Fällen. Das von ihm zitierte Werk mit mindestens 22 Büchern kann demnach, wie ähnlich schon Reti feststellte, nicht identisch sein mit dem Codex Madrid I, sondern entspricht einer bislang verlorenen, stärker systematisierten Überarbeitung der Zeit zwischen 1495 und 1499. Mit großer Wahrscheinlichkeit bezieht sich auch die besprochene Bemerkung zum Anfang eines Buches über Stützen (Madrid I, fol. 171r ,principio del libro“) auf die verlorene systematisierte Neubearbeitung.

In diese Elementi macchinali übernommen waren insbesondere (ab fol. 191r rückläufig bis fol. 177v) die sehr zahlreichen Merksätze, die im Codex Madrid I mit einer fein durchgestrichenen Klammer versehen sind. Reti hat dieses Verfahren bereits näher geschildert. ${ }^{69}$ Für eine Übernahme kommen auch Aussagen zur Statik in Frage, die Leonardo im ersten Teil des Codex Madrid I aus Zeichnungen einer Seite ableitet, am oberen Rand nachträglich ausformuliert und dann mit einer Klammer markiert hat, wie etwa fol. $50 \mathrm{v}$, 75v, 76v, 77v und 78r. All diese Stellen sind in der Edition Retis durch seitliche Klammern markiert.

Wie für Leonardos Studien zur Hydrologie und zu den Möglichkeiten des Fliegens, die er in immer neuen Ansätzen bearbeitet hat, ${ }^{70}$ erhalten wir durch die hier nachgewiesenen verlorenen Schriften auch zu den mechanischen Studien eine klare Etappenfolge seines Bemühens um ständige Verbesserung und inhaltliche Erweiterung. Die Kurzzitate im Codex Madrid I (Teorica) und die späteren Hinweise auf die Elementi macchinali richten sich nicht länger wie in der bisherigen Forschung auf das gleiche verlorene Werk; es sind zwei verschiedene Werke, eines vor Madrid I, das andere nach ihm.

Arbeitete Leonardo somit während der 1490er Jahre in Mailand nacheinander an mindestens drei verschiedenen Werken über Mechanik, zwei systematischen, die beide verloren sind, und einem weiteren in zwei Teilen zu je 95 Blättern, dem erhaltenen Codex Madrid I, so wird verständlich, dass er in derselben Zeit als Maler weniger in Erscheinung trat; nur das Abendmahl in S. Maria delle Grazie, Mailand, fällt als großes Werk in diese Zeit und kam langsam genug voran. Als wesentliche Belastung kam noch die umfangrei-

69 Reti (wie Anm. 7), Bd. 3, S. 69. Weitere Klammern zu theoretischen Aussagen finden sich auf fol. 50v-51v, sie sind dort nicht durchgestrichen. Im Abschnitt fol. 191r-177v bestehen Übereinstimmungen mit Paris Ms. A; vgl. Anna Maria Brizio, Correlazioni e rispondenze fra il Ms. 8937 della Biblioteca Nacional di Madrid e il Ms. A dell'Institut de France, in: L'Arte, 1968, S. 106-111. Die mechanischen Abschnitte des Codex Arundel (ed. Carlo Pedretti/Carlo Vecce, Florenz 1998) konnten noch nicht eingeordnet werden, sie stammen teilweise aus der Zeit vor Madrid I, größerenteils aus den Jahren danach. Eine Zählung, die eine Identifizierung mit Teilen der Elementi macchinali herzustellen erlauben könnte, fand sich bisher auch im Codex Arundel nicht. 
che Planung der großen Reiterstatue für Herzog Francesco Sforza hinzu. Auch in diesem Zusammenhang spielten mechanische Überlegungen, wie jüngst Andrea Bernardoni im Anschluss an Codex Madrid II, fol. 151-157 zeigen konnte, ${ }^{71}$ eine wesentliche Rolle.

Als zumindest hypothetische Erklärung für die deutliche Bevorzugung der Mechanik in den 1490er Jahren wäre in Erwägung zu ziehen, dass am Ende des voraufgehenden Jahrzehnts (Ms. Paris B, ca. 1487-1490) Leonardos frühe Bemühungen um eine Flugmaschine noch stärker im Mittelpunkt standen. Als sich für ihn abklärte, dass die menschliche Muskelkraft zum Antrieb solcher Maschinen nicht ausreichen würde, wandte sich Leonardo in den 1490er Jahren verstärkt den allgemeinen Grundlagen der Mechanik zu und kam erst am Anfang des neuen Jahrhunderts, nach intensiver Beobachtung des Vogelflugs, auf die Flugapparate zurück (Ms. Turin, ca. 1505).

Anschrift des Verfassers: Professor Dr. Dietrich Lohrmann, RWTH Aachen Historisches Institut, Theaterplatz 14, 52062 Aachen. E-mail:

Lohrmann@rwth-aachen.de

71 Vgl. dazu Andrea Bernardoni, Leonardo e il monumento equestre a Francesco Sforza, Florenz 2007. 
